\title{
Noncommutative Burkholder/Rosenthal inequalities associated with convex functions
}

\author{
Narcisse Randrianantoanina ${ }^{\mathrm{a}}$ and Lian $\mathrm{Wu}^{\mathrm{a}, \mathrm{b}, 1}$ \\ a Department of Mathematics, Miami University, Oxford, Ohio 45056,USA. E-mail: randrin@miamioh.edu; wulian@csu.edu.cn \\ ${ }^{\mathrm{b}}$ School of Mathematics and Statistics, Central South University, Changsha 410085, China
}

Received 10 November 2015; revised 2 April 2016; accepted 9 May 2016

\begin{abstract}
We prove noncommutative martingale inequalities associated with convex functions. More precisely, we obtain $\Phi$ moment analogues of the noncommutative Burkholder inequalities and the noncommutative Rosenthal inequalities for any convex Orlicz function $\Phi$ whose Matuzewska-Orlicz indices $p_{\Phi}$ and $q_{\Phi}$ are such that $1<p_{\Phi} \leq q_{\Phi}<2$ or $2<p_{\Phi} \leq q_{\Phi}<\infty$. These results generalize the noncommutative Burkholder/Rosenthal inequalities due to Junge and $\mathrm{Xu}$. The key ingredient in our approach is a simultaneous version of the Burkholder inequality recently proved for the case of noncommutative $L_{p}$-spaces with $1<p<2$.

Résumé. Nous prouvons des inégalités de martingales non commutatives associées à des fonctions convexes. Plus précisément, nous obtenons des analogues des inégalités de Burkholder non commutatives et des inégalités de Rosenthal non commutatives pour des $\Phi$-moments associés à toute fonction convexe $\Phi$ dont les indices de Matuzewska-Orlicz $p_{\Phi}$ et $q_{\Phi}$ vérifient $1<p_{\Phi} \leq q_{\Phi}<2$ ou $2<p_{\Phi} \leq q_{\Phi}<\infty$. Ces résultats généralisent les inégalités de Burkholder/Rosenthal non commutatives obtenues par Junge et Xu. L'ingrédient clé de notre approche est une version simultanée de l'inégalité de Burkholder récemment demontrée dans le cas des espaces $L_{p}$ non commutatifs pour $1<p<2$.
\end{abstract}

MSC: Primary 46L53; 46L52; secondary 47L05; 60G42

Keywords: Noncommutative Burkholder inequalities; Noncommutative Rosenthal inequalities; Orlicz functions; Moment inequalities; Interpolations

\section{Introduction}

The theory of noncommutative martingales has enjoyed considerable progress in recent years due to its interaction with other field of mathematics such as operator spaces and free probability. Many classical martingale inequalities have been extended to the noncommutative setting. We refer to [22-24,38,40,42] and the references therein for more information on noncommutative martingales. This paper deals with moment inequalities associated with convex functions for noncommutative martingales.

The study of convex function inequalities for martingales was initiated by Burkholder and Gundy in their seminal paper [8]. The general theme of their work can be summarized as follows: let $\mathfrak{M}$ be a family of martingales on a probability space $(\Omega, \Sigma, \mathbb{P})$ and $\Phi$ be a nonnegative and increasing convex function on $[0, \infty)$ satisfying $\Phi(0)=0$ and $\lim _{t \rightarrow \infty} \Phi(t)=\infty$. If $U$ and $V$ are operators on $\mathfrak{M}$ with values in the set of nonnegative random variables on $(\Omega, \Sigma, \mathbb{P})$, under what conditions on $\Phi$ and $\mathfrak{M}$ does the inequality $\mathbb{E}[\Phi(V f)] \leq C \mathbb{E}[\Phi(U f)]$ hold for all martingales $f \in \mathfrak{M}$. For the special case where $\Phi(t)=t^{p}$ for $1 \leq p<\infty$, the above question reduces to comparisons of $p$ th moments of the nonnegative random variables $V f$ and $U f$. For general convex function $\Phi$ satisfying the conditions

\footnotetext{
${ }^{1}$ Supported in part by NSFC (No. 11471337) and the China Scholarship Council.
} 
described above, these types of inequalities are generally referred to as $\Phi$-moment inequalities. Typical examples of such operators $U$ and $V$ are, among others, square functions, maximal functions, martingale transforms, ect. Subsequently, many classical $p$ th moment inequalities for martingales were extended to convex function inequalities. We refer to $[6,7,17]$ for more information on the development of $\Phi$-moment inequalities from the classical martingale theory.

Recently, several $\Phi$-moment inequalities have been extended to the context of noncommutative martingales. This was initiated by Bekjan and Chen in [1]. For instance, $\Phi$-moment versions of the noncommutative BurkholderGundy inequalities from [40] were treated in [1] (see also the work of Dirksen and Ricard [13]). Various maximal type-inequalities for noncommutative martingales initially proved in [20] for the case of noncommutative $L_{p}$-spaces are now known to be valid for a wider class of convex functions $([2,11])$. In this paper, we are mainly interested on inequalities involving conditioned square functions of noncommutative martingales. To better explain our motivation and results, let us begin by recalling Rosenthal's remarkable inequalities ([46]) which state that if $2 \leq p<\infty$ and $\left(g_{n}\right)_{n \geq 1}$ is a sequence of independent mean-zero random variables in $L_{p}(\Omega, \Sigma, \mathbb{P})$, then the following holds:

$$
\left(\mathbb{E}\left|\sum_{n \geq 1} g_{n}\right|^{p}\right)^{1 / p} \simeq{ }_{p}\left(\sum_{n \geq 1} \mathbb{E}\left|g_{n}\right|^{2}\right)^{1 / 2}+\left(\sum_{n \geq 1} \mathbb{E}\left|g_{n}\right|^{p}\right)^{1 / p},
$$

where $\simeq_{p}$ means equivalence of norms up to constants depending only on $p$. The equivalence (1.1) was initially established in order to construct some new classes of Banach spaces but over the years it has been proven to have many applications in other areas of mathematics. The martingale version of (1.1) was discovered almost simultaneously by Burkholder in [6]. In fact, a $\Phi$-moment version was obtained by Burkholder that takes the following form: if $\Phi$ is a convex Orlicz function on $[0, \infty)$ that satisfies the so called $\Delta_{2}$-condition then for any martingale $f=\left(f_{n}\right)_{n \geq 1}$ adapted to a given filtration $\left\{\Sigma_{n}\right\}_{n \geq 1}$ of $\sigma$-subalgebras of $\Sigma$ satisfying $\sigma\left(\bigcup_{n \geq 1} \Sigma_{n}\right)=\Sigma$, the following holds (here, we use the convention that $\Sigma_{0}=\Sigma_{1}$ ):

$$
\sup _{n \geq 1} \mathbb{E}\left[\Phi\left(\left|f_{n}\right|\right)\right] \leq C_{\Phi} \mathbb{E}[\Phi(s(f))]+\mathbb{E}\left[\Phi\left(d^{*}\right)\right]
$$

where $s(f)=\left(\sum_{n \geq 1} \mathbb{E}\left[\left|d f_{n}\right|^{2} \mid \Sigma_{n-1}\right]\right)^{1 / 2}$ is the conditioned square function of the martingale $f$ while $d^{*}=$ $\sup _{n \geq 1}\left|d f_{n}\right|$ is the maximal function of its martingale difference sequence. On the other hand, noncommutative analogues of the Burkholder/Rosenthal inequalities for the case of $p$ th moments have been discovered by Junge and Xu in $[23,25]$. More precisely, they obtained that if $2 \leq p<\infty$ and $x=\left(x_{n}\right)_{n \geq 1}$ is a noncommutative martingale that is $L_{p}$-bounded then

$$
\|x\|_{p} \simeq_{p} \max \left\{\left\|s_{c}(x)\right\|_{p},\left\|s_{r}(x)\right\|_{p},\left(\sum_{n \geq 1}\left\|d x_{n}\right\|_{p}^{p}\right)^{1 / p}\right\},
$$

where $s_{c}(x)$ and $s_{r}(x)$ denote the column version and the row version of conditioned square functions which we refer to the next section for formal definitions. In addition, they also managed to formulate and prove the corresponding inequalities for the range $1<p<2$ which are dual to (1.3) that can be roughly stated as follows: if $x=\left(x_{n}\right)_{n \geq 1}$ is a noncommutative martingale in $L_{2}(\mathcal{M})$ then

$$
\|x\|_{p} \simeq_{p} \inf \left\{\left\|s_{c}(y)\right\|_{p}+\left\|s_{r}(z)\right\|_{p}+\left(\sum_{n \geq 1}\left\|d w_{n}\right\|_{p}^{p}\right)^{1 / p}\right\},
$$

where the infimum is taken over all decompositions $x=y+z+w$ with $y, z$, and $w$ are martingales. Reasons behind the fact that the two cases $1<p<2$ and $2 \leq p<\infty$ have to be different are now well-understood in the field. As shown in $[23,25]$, the equivalences (1.3) and (1.4) have far reaching applications ranging from random matrices to operator space classifications of some classes of subspaces of noncomutative $L_{p}$-spaces. We should also mention that a recent work of Junge and Zeng on improved constants on the noncommutative Rosenthal inequalities [26] have applications in compressed sensing. Recently, equivalences (1.3) and (1.4) were extended to certain classes of martingales in noncommutative symmetric spaces for which we refer to [45] for details. Motivated by these various results, we 
consider $\Phi$-moments of conditioned square functions of noncommutative martingales in the spirit of (1.2). We obtain natural extensions of the noncommutative Burkholder inequalities (1.3) and (1.4). We work with semifinite von Neumann algebra equipped with normal semifinite faithful trace $(\mathcal{M}, \tau)$. In formulating the right versions of $\Phi$-moments, one needs to consider martingales that are bounded in the noncommutative Orlicz space $L_{\Phi}(\mathcal{M})$. In addition, we also require some conditions on the lower and upper Matuzewska-Orlicz indices $p_{\Phi}$ and $q_{\Phi}$ of the convex function $\Phi$ which in some sense mimic the role of the index $p$ in the noncommutative Burkholder/Rosenthal inequalities. Our principal results may be viewed as common generalizations of (1.2), (1.3), and (1.4). We may summarize these results as follow:

If $2<p_{\Phi} \leq q_{\Phi}<\infty$, then for any $L_{\Phi}(\mathcal{M})$-bounded martingale $x=\left(x_{n}\right)_{n \geq 1}$,

$$
\sup _{n \geq 1} \tau\left[\Phi\left(\left|x_{n}\right|\right)\right] \simeq_{\Phi} \max \left\{\tau\left[\Phi\left(s_{c}(x)\right)\right], \tau\left[\Phi\left(s_{r}(x)\right)\right], \sum_{n \geq 1} \tau\left[\Phi\left(\left|d x_{n}\right|\right)\right]\right\} .
$$

If $1<p_{\Phi} \leq q_{\Phi}<2$, then for any $L_{\Phi}(\mathcal{M})$-bounded martingale $x=\left(x_{n}\right)_{n \geq 1}$,

$$
\sup _{n \geq 1} \tau\left[\Phi\left(\left|x_{n}\right|\right)\right] \simeq_{\Phi} \inf \left\{\tau\left[\Phi\left(s_{c}(y)\right)\right]+\tau\left[\Phi\left(s_{r}(z)\right)\right]+\sum_{n \geq 1} \tau\left[\Phi\left(\left|d w_{n}\right|\right)\right]\right\},
$$

where the infimum is taken over all decompositions $x=y+z+w$ with $y, z$, and $w$ are martingales. We refer to Theorem 4.1 and Theorem 4.4 for more detailed explanations of the notation used in the formulations of (1.5) and (1.6). These results complement the series of $\Phi$-moment inequalities from $[1,2,11,13]$. We note that if $\Phi(t)=t^{p}$ for $1<p<\infty$, then these results become exactly the Junge and Xu's noncommutative Burkholder inequalities. It is also important to note that the case of noncommutative symmetric spaces treated in [45] does not imply the corresponding $\Phi$-moment inequalities.

The original proof of (1.2) was primarily based on careful analysis of distribution functions which heavily relied on stopping times and the so-called good $\lambda$-inequalities. Stopping times and good $\lambda$-inequalities are very powerful techniques in the classical setting. Unfortunately, these techniques are not available in the noncommutative setting. Therefore, our method of proof has to rely on new ideas. Our approach was primarily motivated by an observation that singular values of measurable operators are closely connected to $K$-functionals from interpolation theory. Our strategy is to focus first on (1.6). As noted earlier, we heavily employ results from interpolation theory. As in the case of noncommutative symmetric spaces, a simultaneous decomposition version of (1.4) from [45] also plays a significant role in our argument. The proof of (1.5) is a duality type-argument. Since $\Phi$-moments are usually not defining a norm, we had to provide the proper connection between any given Orlicz function and its complementary that is suitable for moment inequalities. This connection appears as an operator equality that may be viewed as operator reverse to the classical Young's inequality. We refer to Proposition 2.3 for the exact statement. We should point out that for the case of square functions, the proofs of the $\Phi$-moment versions of the noncommutative Burkholder-Gundy in $[1,13,18]$ depend on some versions of $\Phi$-moment extensions of the noncommutative Khintchine inequalities.

The paper is structured as follows. In Section 2, we setup some basic notation and present some preliminary results concerning noncommutative Orlicz spaces and noncommutative martingales. We review the constructions leading up to all relevant Hardy type spaces that we need for our presentation. In Section 3, we isolate and prove some key inequalities involving $\Phi$-moments, $K$-functionals, and $J$-functionals from interpolation theory. Section 4 is devoted to the statements and proofs of our $\Phi$-moment versions of the noncommutative Burkholder inequalities. In Section 5, we examine the case of sums of noncommuting independent sequences of mean zero in the sense of [25]. In particular, we provide $\Phi$-moment analogues of the noncommutative Rosenthal inequalities from [25]. We also provide the corresponding Rosenthal inequalities for noncommuting independent sequences in noncommutative symmetric spaces. In the last section, we discuss possible future direction for general $\Phi$-moments and list some related open problems. 


\section{Preliminaries}

\subsection{Orlicz functions and noncommutative Orlicz spaces}

Throughout this paper, $\mathcal{M}$ will always denote a semifinite von Neumann algebra equipped with a faithful normal semifinite trace $\tau$. Assume that $\mathcal{M}$ is acting on a Hilbert space $H$. A closed densely defined operator $x$ on $H$ is said to be affiliated with $\mathcal{M}$ if $x$ commutes with every unitary $u$ in the commutant $\mathcal{M}^{\prime}$ of $\mathcal{M}$. If $a$ is a densely defined self-adjoint operator on $H$ and $a=\int_{-\infty}^{\infty} s d e_{s}^{a}$ is its spectral decomposition, then for any Borel subset $B \subseteq \mathbb{R}$, we denote by $\chi_{B}(a)$ the corresponding spectral projection $\int_{-\infty}^{\infty} \chi_{B}(s) d e_{s}^{a}$. An operator $x$ affiliated with $\mathcal{M}$ is called $\tau$ measurable if there exists $s>0$ such that $\tau\left(\chi_{(s, \infty)}(|x|)\right)<\infty$. It is known that the set of all $\tau$-measurable operators with respect to $(\mathcal{M}, \tau)$ is a topological $*$-algebra which we will denote by $L_{0}(\mathcal{M}, \tau)$. We refer to $[37,41,48]$ for unexplained terminology. For $x \in L_{0}(\mathcal{M}, \tau)$, define the distribution function of $x$ by setting for $s>0$,

$$
\lambda_{s}(x)=\tau(\chi(s, \infty)(|x|)) .
$$

The generalized singular value of $x$ is defined by

$$
\mu_{t}(x)=\inf \left\{s>0 ; \lambda_{s}(x) \leq t\right\}, \quad t>0 .
$$

The function $t \mapsto \mu_{t}(x)$ from $(0, \infty)$ into [0, $)$ is right-continuous and nonincreasing ([16]). We note that for the case where $\mathcal{M}$ is the abelian von Neumann algebra $L_{\infty}(0, \infty)$ with the trace given by integration with respect to the Lebesgue measure, $L_{0}(\mathcal{M}, \tau)$ becomes the linear space of all measurable functions $L_{0}(0, \infty)$ and $\mu(f)$ is the decreasing rearrangement of the function $|f|$ in the sense of [31].

By an Orlicz function $\Phi$ on $[0, \infty)$, we mean a continuous, increasing, and convex function such that $\Phi(0)=0$ and $\lim _{t \rightarrow \infty} \Phi(t)=\infty$. For examples and basic properties of Orlicz functions we refer to [29,34,35].

Given an operator $x \in L_{0}(\mathcal{M}, \tau)$ and an Orlicz function $\Phi$, we may define $\Phi(|x|)$ through functional calculus. That is, if $|x|=\int_{0}^{\infty} s d e_{s}^{|x|}$ is the spectral decomposition of $|x|$, then

$$
\Phi(|x|)=\int_{0}^{\infty} \Phi(s) d e_{s}^{|x|} .
$$

The operator $\Phi(|x|)$ is then a positive $\tau$-measurable operator. It is important to observe that the trace of $\Phi(|x|)$ can be calculated using either the distribution function of $|x|$ or the singular value function of $|x|$. Indeed, one can easily deduce from [16, Corollary 2.8] that if $x \in L_{0}(\mathcal{M}, \tau)$, then we have the identities:

$$
\tau[\Phi(|x|)]=\int_{0}^{\infty} \lambda_{s}(|x|) d \Phi(s)=\int_{0}^{\infty} \Phi\left(\mu_{t}(x)\right) d t .
$$

The quantity $\tau[\Phi(|x|)]$ will be referred to as the $\Phi$-moment of the operator $|x|$. Clearly, if we consider the power function $\Phi(t)=t^{p}$ for $1 \leq p<\infty$, then this reduces to the usual notion of $p$ th moment of $|x|$. It is however important to point out that in general $\Phi$-moments do not necessarily define a norm and therefore many tools used for various results on $p$ th moments are no longer available when dealing with $\Phi$-moments.

We will assume throughout that $\Phi$ satisfies a growth condition known as the $\Delta_{2}$-condition. That is, for some constant $C>0$,

$$
\Phi(2 t) \leq C \Phi(t), \quad t \geq 0
$$

It is easy to check that $\Phi$ satisfies the $\Delta_{2}$-condition if and only if for every $a>0$, there exists a constant $C_{a}>0$ such that $\Phi(a t) \leq C_{a} \Phi(t)$ for all $t>0$. More generally, by functional calculus, if $0 \leq x \in L_{0}(\mathcal{M}, \tau)$ and $a$ is a positive scalar then the following operator inequality holds:

$$
\Phi(a x) \leq C_{a} \Phi(x) .
$$


One can also deduce from the integral representation stated above and [16, Theorem 4.4(iii)] that if $\left(x_{i}\right)_{i=1}^{n}$ is a finite sequence in $L_{0}(\mathcal{M})$ and $\left(\alpha_{i}\right)_{i=1}^{n} \subset(0,1)^{n}$ with $\sum_{i=1}^{n} \alpha_{i}=1$ then

$$
\tau\left[\Phi\left(\left|\sum_{i=1}^{n} \alpha_{i} x_{i}\right|\right)\right] \leq \sum_{i=1}^{n} \alpha_{i} \tau\left[\Phi\left(\left|x_{i}\right|\right)\right] .
$$

As a consequence of (2.1) and the $\Delta_{2}$-condition, we have the quasi-triangle inequality:

$$
\tau[\Phi(|x+y|)] \leq C_{\Phi}(\tau[\Phi(|x|)]+\tau[\Phi(|y|)]) .
$$

These inequalities will be used repeatedly throughout. Next, we introduce some standard indices for Orlicz functions. For a given Orlicz function $\Phi$, we let

$$
M(t, \Phi)=\sup _{s>0} \frac{\Phi(t s)}{\Phi(s)}, \quad t>0,
$$

and

$$
p_{\Phi}=\lim _{t \rightarrow 0^{+}} \frac{\log (M(t, \Phi))}{\log t}, \quad q_{\Phi}=\lim _{t \rightarrow \infty} \frac{\log (M(t, \Phi))}{\log t} .
$$

The two quantities $p_{\Phi}$ and $q_{\Phi}$ are known as Matuzewska-Orlicz indices of the Orlicz function $\Phi$. For more information on these indices and their connections with other indices, we refer to the monographs [34,35]. In general, we have $1 \leq p_{\Phi} \leq q_{\Phi} \leq \infty$ and the $\Delta_{2}$-condition is equivalent to $q_{\Phi}<\infty$.

We now recall the definition of Orlicz spaces. For a given Orlicz function $\Phi$, the Orlicz function space $L_{\Phi}(0, \infty)$ is the set of all Lebesgue measurable functions $f$ defined on $(0, \infty)$ such that for some constant $c>0$,

$$
\int_{0}^{\infty} \Phi(|f(t)| / c) d t<\infty
$$

If we equip $L_{\Phi}(0, \infty)$ with the Luxemburg norm:

$$
\|f\|_{L_{\Phi}}=\inf \left\{c>0: \int_{0}^{\infty} \Phi(|f(t)| / c) d t \leq 1\right\},
$$

then $L_{\Phi}(0, \infty)$ is a fully symmetric Banach function space in the sense of [15]. Moreover, the Boyd indices of $L_{\Phi}(0, \infty)$ coincide with the indices $p_{\Phi}$ and $q_{\Phi}$ (see [34]). We may define the noncommutative Orlicz space $L_{\Phi}(\mathcal{M}, \tau)$ following the general scheme of constructing noncommutative analogue of symmetric function spaces as described in $[14,15,28,49]$. Note that under the $\Delta_{2}$-condition, $x \in L_{\Phi}(\mathcal{M}, \tau)$ if and only if $\tau[\Phi(|x|)]<\infty$. Also, it is clear that if $\Phi(t)=t^{p}$ with $1 \leq p<\infty$, then $L_{\Phi}(\mathcal{M}, \tau)=L_{p}(\mathcal{M}, \tau)$ where $L_{p}(\mathcal{M}, \tau)$ is the usual noncommutative $L_{p}$-space associated with $(\mathcal{M}, \tau)$.

We now gather some preliminary results on noncommutative Orlicz spaces that we will need in the sequel. We assume that the next lemma is known but we could not find any specific reference. We feel that a proof is needed since in general $\Phi$-moments do not define a norm.

Lemma 2.1. Let $\left(x_{n}\right)_{n \geq 1}$ be a sequence in $L_{\Phi}(\mathcal{M}, \tau)$ and $x \in L_{\Phi}(\mathcal{M}, \tau)$.

(i) If $\lim _{n \rightarrow \infty}\left\|x_{n}-x\right\|_{L_{\Phi}(\mathcal{M})}=0$ then $\lim _{n \rightarrow \infty} \tau\left[\Phi\left(\left|x_{n}\right|\right)\right]=\tau[\Phi(|x|)]$

(ii) If $\left(x_{n}\right)_{n \geq 1}$ converges to $x$ weakly in $L_{\Phi}(\mathcal{M}, \tau)$ then $\tau[\Phi(|x|)] \leq \liminf _{n \rightarrow \infty} \tau\left[\Phi\left(\left|x_{n}\right|\right)\right]$.

Proof. Let us begin with the first item. Recall that since $\Phi$ satisfies the $\Delta_{2}$-condition, a sequence $\left(f_{n}\right)_{n \geq 1}$ in $L_{\Phi}$ converges in norm to $f$ in $L_{\Phi}$ if and only if $\lim _{n \rightarrow \infty} \int_{0}^{\infty} \Phi\left(\left|f_{n}(t)-f(t)\right|\right) d t=0$. Therefore, $\lim _{n \rightarrow \infty}\left\|x_{n}-x\right\|_{L_{\Phi}(\mathcal{M})}=$ 0 if and only if $\lim _{n \rightarrow \infty} \int_{0}^{\infty} \Phi\left(\mu_{t}\left(x_{n}-x\right)\right) d t=0$. We have from [14, Theorem 3.4] that for every $n \geq 1$, the function $\left|\mu\left(x_{n}\right)-\mu(x)\right|$ is submajorized by $\mu\left(x_{n}-x\right)$ in the sense that for every $t>0$,

$$
\int_{0}^{t}\left|\mu_{s}\left(x_{n}\right)-\mu_{s}(x)\right| d s \leq \int_{0}^{t} \mu_{s}\left(x_{n}-x\right) d s .
$$


Since $L_{\Phi}(0, \infty)$ is fully symmetric, it follows that $\lim _{n \rightarrow \infty}\left\|\mu\left(x_{n}\right)-\mu(x)\right\|_{L_{\Phi}}=0$. Next, we observe that $\left\{\Phi\left(\mu\left(x_{n}\right)\right) ; n \geq 1\right\}$ is a uniformly integrable subset of $L_{1}(0, \infty)$. This is the case since by the $\Delta_{2}$-condition, there is a constant $C_{\Phi}$ so that for every $n \geq 1$, we have $\Phi\left(\mu\left(x_{n}\right)\right) \leq C_{\Phi} \Phi\left(\left|\mu\left(x_{n}\right)-\mu(x)\right|\right)+C_{\Phi} \Phi(\mu(x))$.

Now, fix an arbitrary subsequence $\left(y_{n}\right)_{n \geq 1}$ of $\left(x_{n}\right)_{n \geq 1}$. There exists a further subsequence $\left(y_{n_{k}}\right)_{k \geq 1}$ of $\left(y_{n}\right)_{n \geq 1}$ so that $\mu\left(y_{n_{k}}\right) \rightarrow \mu(x)$ a.e. By uniform integrability of $\left\{\Phi\left(\mu\left(x_{n}\right)\right) ; n \geq 1\right\}$, we have

$$
\lim _{k \rightarrow \infty} \int_{0}^{\infty} \Phi\left(\mu_{t}\left(y_{n_{k}}\right)\right) d t=\int_{0}^{\infty} \Phi\left(\mu_{t}(x)\right) d t
$$

This is equivalent to $\lim _{k \rightarrow \infty} \tau\left[\Phi\left(\left|y_{n_{k}}\right|\right)\right]=\tau[\Phi(|x|)]$. Therefore, we have shown that every subsequence of $\left\{\tau\left[\Phi\left(\left|x_{n}\right|\right)\right]\right\}_{n \geq 1}$ has further subsequences that converge to $\tau[\Phi(|x|)]$. This shows that $\lim _{n \rightarrow \infty} \tau\left[\Phi\left(\left|x_{n}\right|\right)\right]=$ $\tau[\Phi(|x|)]$ as claimed.

For the second item, assume now that $x_{n} \rightarrow x$ weakly and let $\xi$ be a limit point of the bounded sequence $\left\{\tau\left[\Phi\left(\left|x_{n}\right|\right)\right]\right\}_{n \geq 1}$. Fix a subsequence $\left(y_{n}\right)$ of $\left(x_{n}\right)$ such that $\xi=\lim _{n \rightarrow \infty} \tau\left[\Phi\left(\left|y_{n}\right|\right)\right]$. Next, we choose a sequence $\left(z_{n}\right)$ consisting of block convex combinations of $\left(y_{n}\right)$ such that $\lim _{n \rightarrow \infty}\left\|z_{n}-x\right\|_{L_{\Phi}(\mathcal{M})}=0$. From the first item, we have $\tau[\Phi(|x|)]=\lim _{n \rightarrow \infty} \tau\left[\Phi\left(\left|z_{n}\right|\right)\right]$. For each $n \geq 1$, write $z_{n}=\sum_{j=p_{n}}^{q_{n}} \alpha_{j} y_{j}$ with $1 \leq p_{1}<q_{1}<p_{2}<q_{2}<\cdots$, $\alpha_{i} \in[0,1]$ for all $i \geq 1$, and $\sum_{i=p_{n}}^{q_{n}} \alpha_{i}=1$ for all $n \geq 1$. It follows from (2.1) that

$$
\begin{aligned}
\tau[\Phi(|x|)] & =\lim _{n \rightarrow \infty} \tau\left[\Phi\left(\left|z_{n}\right|\right)\right] \\
& \leq \lim _{n \rightarrow \infty} \sum_{i=p_{n}}^{q_{n}} \alpha_{i} \tau\left[\Phi\left(\left|y_{i}\right|\right)\right] \\
& =\lim _{n \rightarrow \infty} \tau\left[\Phi\left(\left|y_{n}\right|\right)\right]=\xi .
\end{aligned}
$$

The desired inequality follows from taking the infimum over all such limit points.

We now discuss some background on complementary Orlicz functions. Let $\Phi$ be an Orlicz function. It is wellknown that $\Phi$ admits an integral representation

$$
\Phi(u)=\int_{0}^{u} \varphi(s) d s, \quad u>0,
$$

where $\varphi$ is a nondecreasing right-continuous function defined on the interval $[0, \infty)$. The function $\varphi$ is usually referred to as the right derivative of $\Phi$. Let $\psi(t)=\sup \{s: \varphi(s) \leq t\}$ be the right inverse of $\varphi$. We observe that $\psi$ is a nondecreasing right-continuous function on $[0, \infty)$ and if $\varphi$ is a continuous function then $\psi$ is the usual inverse of $\varphi$. We define the Orlicz complementary function to $\Phi$ by setting:

$$
\Phi^{*}(v)=\int_{0}^{v} \psi(t) d t, \quad v>0 .
$$

Clearly, $\Phi^{*}$ is an Orlicz function and under some natural conditions on $\Phi$, there is a canonical duality between the noncommutative Orlicz spaces $L_{\Phi}(\mathcal{M}, \tau)$ and $L_{\Phi^{*}}(\mathcal{M}, \tau)$. We refer to [35, Chapter 9] for more detailed accounts of such duality in the commutative case. It is worth mentioning that for the special case where $\Phi(u)=u^{r} / r$ for some $1<r<\infty$ then $\Phi^{*}(v)=v^{r^{\prime}} / r^{\prime}$ where $r^{\prime}$ denotes the index conjugate to $r$. Therefore, we may view $\Phi^{*}$ as the Orlicz function analogue of the concept of conjugate indices. In fact, from [35, Corollary 11.6], the indices of $\Phi^{*}$ satisfy:

$$
1 / p_{\Phi}+1 / q_{\Phi^{*}}=1 / p_{\Phi^{*}}+1 / q_{\Phi}=1 .
$$

We refer to [29, Chap. I] for more in depth discussion on connections between $\Phi$ and $\Phi^{*}$. Another fact that is of particular importance for our purpose is the so-called Young's inequality which states that for every $u, v \geq 0$, the following inequality holds:

$$
u v \leq \Phi(u)+\Phi^{*}(v) .
$$


As an elementary application of Young's inequality, we record the following lemma for further use.

Lemma 2.2. For every $x \in L_{\Phi}(\mathcal{M})$ and $y \in L_{\Phi^{*}}(\mathcal{M}), x y \in L_{1}(\mathcal{M})$ and

$$
\|x y\|_{1} \leq \tau[\Phi(|x|)]+\tau\left[\Phi^{*}(|y|)\right] .
$$

Proof. First, we note from basic properties of generalized singular values that if $x y \in L_{1}(\mathcal{M})$ then using properties of singular values ([16, Theorem 4.2]),

$$
\|x y\|_{1}=\int_{0}^{\infty} \mu_{t}(x y) d t \leq \int_{0}^{\infty} \mu_{t}(x) \mu_{t}(y) d t .
$$

By Young's inequality, we deduce that

$$
\|x y\|_{1} \leq \int_{0}^{\infty} \Phi\left(\mu_{t}(x)\right) d t+\int_{0}^{\infty} \Phi^{*}\left(\mu_{t}(y)\right) d t,
$$

which is clearly the desired inequality.

Our next result may be viewed as an operator reverse Young's inequality and could be of independent interest.

Proposition 2.3. Let $\Phi$ be an Orlicz function with $1<p_{\Phi} \leq q_{\Phi}<\infty$. For every $0 \leq x \in L_{\Phi}(\mathcal{M})$ there exists $0 \leq$ $y \in L_{\Phi^{*}}(\mathcal{M})$ such that $y$ commutes with $x$ and satisfies

$$
x y=\Phi(x)+\Phi^{*}(y) .
$$

Proof. We note first that since $p_{\Phi}>1$, we have $q_{\Phi^{*}}<\infty$ and therefore $\Phi^{*}$ satisfies the $\Delta_{2}$-condition. Let $\varphi$ denote the right derivative of $\Phi$. The proposition is a consequence of the following fact which can be found in [29, p. 13] (see also [35, p. 48]):

$$
u v=\Phi(u)+\Phi^{*}(v) \quad \Longleftrightarrow \quad v=\varphi(u) .
$$

That is, at the function level, the following identity holds:

$$
u \varphi(u)=\Phi(u)+\Phi^{*}(\varphi(u)), \quad u \geq 0 .
$$

We remark that since the function $\varphi$ is monotone, it is Borel measurable. Using functional calculus on the positive operator $x$, the preceding identity yields:

$$
x \varphi(x)=\Phi(x)+\Phi^{*}(\varphi(x)) .
$$

It is enough to consider $y=\varphi(x)$. Clearly, $y \geq 0$ and commutes with $x$. To verify that $y \in L_{\Phi^{*}}(\mathcal{M})$, we appeal to another index of $\Phi$ defined as follows:

$$
b_{\Phi}:=\sup _{t>0} \frac{t \Phi^{\prime}(t)}{\Phi(t)}=\sup _{t>0} \frac{t \varphi(t)}{\Phi(t)} .
$$

In general, we only have $q_{\Phi} \leq b_{\Phi}$ but the relevant property we need is that the $\Delta_{2}$-condition is equivalent to $b_{\Phi}<\infty$. These facts were taken from [34, Theorem 3.2]. The crucial observation we make is that for every $t>0$,

$$
t \varphi(t) \leq b_{\Phi} \Phi(t)
$$

where the function on the right hand side is finite for all $t>0$. Thus, by functional calculus and the definition of $y$, the preceding inequality yields the operator inequality:

$$
0 \leq x y \leq b_{\Phi} \Phi(x) .
$$


This is equivalent to $\Phi^{*}(y) \leq\left(b_{\Phi}-1\right) \Phi(x)$. Taking traces, we have

$$
\tau\left[\Phi^{*}(y)\right] \leq\left(b_{\Phi}-1\right) \tau[\Phi(x)] .
$$

Since $x \in L_{\Phi}(\mathcal{M})$, the right hand side is finite and therefore, we have $\tau\left[\Phi^{*}(y)\right]<\infty$. As $\Phi^{*}$ satisfies the $\Delta_{2^{-}}$ condition, this is equivalent to $y \in L_{\Phi^{*}}(\mathcal{M})$. The proof is complete.

\subsection{Noncommutative martingales}

Let us now review the general setup for noncommutative martingales. For simplicity, we assume for the remaining of the paper that $\mathcal{M}_{*}$ is separable. In the sequel, we always denote by $\left(\mathcal{M}_{n}\right)_{n \geq 1}$ an increasing sequence of von Neumann subalgebras of $\mathcal{M}$ whose union is weak*-dense in $\mathcal{M}$. For $n \geq 1$, we assume that there exists a trace preserving conditional expectation $\mathcal{E}_{n}$ from $\mathcal{M}$ onto $\mathcal{M}_{n}$. It is well-known that for $1 \leq p \leq \infty, \mathcal{E}_{n}$ extends to a contractive projection from $L_{p}(\mathcal{M}, \tau)$ onto $L_{p}\left(\mathcal{M}_{n}, \tau_{n}\right)$, where $\tau_{n}$ denotes the restriction of $\tau$ on $\mathcal{M}_{n}$. More generally, if $\Phi$ is an Orlicz function, then since $L_{\Phi}(0, \infty)$ is fully symmetric, it follows that $\mathcal{E}_{n}$ is a contractive projection from $L_{\Phi}(\mathcal{M}, \tau)$ onto $L_{\Phi}\left(\mathcal{M}_{n}, \tau_{n}\right)$ (see for instance, [12, Proposition 2.1]).

Definition 2.4. A sequence $x=\left(x_{n}\right)_{n \geq 1}$ in $L_{1}(\mathcal{M})+\mathcal{M}$ is called a noncommutative martingale with respect to $\left(\mathcal{M}_{n}\right)_{n \geq 1}$ if $\mathcal{E}_{n}\left(x_{n+1}\right)=x_{n}$ for every $n \geq 1$.

If in addition, all $x_{n}$ 's belong to $L_{\Phi}(\mathcal{M})$ for a given Orlicz function $\Phi$, then $x$ is called an $L_{\Phi}(\mathcal{M})$-martingale. In this case, we may define

$$
\|x\|_{L_{\Phi}(\mathcal{M})}=\sup _{n \geq 1}\left\|x_{n}\right\|_{L_{\Phi}(\mathcal{M})}
$$

For the case where $\|x\|_{L_{\Phi}(\mathcal{M})}<\infty$, then $x$ is called a bounded $L_{\Phi}(\mathcal{M})$-martingale. We note that if the indices of $\Phi$ satisfy $1<p_{\Phi} \leq q_{\Phi}<\infty$, then $L_{\Phi}(\mathcal{M})$ is a reflexive space. In this case, any bounded $L_{\Phi}(\mathcal{M})$-martingale $\left(x_{n}\right)_{n \geq 1}$ converges to some $x_{\infty}$ in $L_{\Phi}(\mathcal{M})$ that satisfies $\mathcal{E}_{n}\left(x_{\infty}\right)=x_{n}$ for all $n \geq 1$. From this fact, whenever $1<p_{\Phi} \leq q_{\Phi}<$ $\infty$, we will not make any distinction between operators in $L_{\Phi}(\mathcal{M})$ and bounded $L_{\Phi}(\mathcal{M})$-martingales.

Let $x=\left(x_{n}\right)_{n \geq 1}$ be a noncommutative martingale with respect to $\left(\mathcal{M}_{n}\right)_{n \geq 1}$. Define $d x_{n}=x_{n}-x_{n-1}$ for $n \geq 1$ with the usual convention that $x_{0}=0$. The sequence $d x=\left(d x_{n}\right)_{n \geq 1}$ is called the martingale difference sequence of $x$.

In this paper, we will be mainly working with conditioned square functions and noncommutative conditioned Hardy spaces. We refer the reader to $[1,45]$ for noncommutative Hardy spaces associated with square functions. Recall that if $x=\left(x_{n}\right)_{n \geq 1}$ is an $L_{2}(\mathcal{M})+\mathcal{M}$-martingale, then we can formally define:

$$
s_{c}(x)=\left(\sum_{k \geq 1} \mathcal{E}_{k-1}\left|d x_{k}\right|^{2}\right)^{1 / 2} \text { and } s_{r}(x)=\left(\sum_{k \geq 1} \mathcal{E}_{k-1}\left|d x_{k}^{*}\right|^{2}\right)^{1 / 2} .
$$

These are called the column and row conditioned square functions of $x$, respectively. We want to emphasize that when $d x_{k} \notin L_{2}(\mathcal{M})+\mathcal{M}$, then $\left|d x_{k}\right|^{2}$ may not be necessary in $L_{1}(\mathcal{M})+\mathcal{M}$. Therefore, $\mathcal{E}_{k-1}\left|d x_{k}\right|^{2}$ is not necessarily a well-defined object. Thus, extra cares are needed for martingales that do not belong to $L_{2}(\mathcal{M})+\mathcal{M}$. Since the main topic of this paper is dealing with various inequalities involving conditioned square functions, we will review the general construction which is based on the so-called conditioned spaces. These were formally introduced by Junge in [20] for noncommutative $L_{p}$-spaces and were extensively used by Junge and $\mathrm{Xu}$ in $[23,25]$. Recently, these ideas were adapted in [45] to the case of more general classes of noncommutative symmetric spaces. Below, we use the usual convention that $\mathcal{E}_{0}=\mathcal{E}_{1}$.

Let $\mathcal{E}: \mathcal{M} \rightarrow \mathcal{N}$ be a normal faithful conditional expectation, where $\mathcal{N}$ is a von Neumann subalgebra of $\mathcal{M}$. For $0<p \leq \infty$, we define the conditioned space $L_{p}^{c}(\mathcal{M}, \mathcal{E})$ to be the completion of $\mathcal{M} \cap L_{p}(\mathcal{M})$ with respect to the (quasi) norm

$$
\|x\|_{L_{p}^{c}(\mathcal{M}, \mathcal{E})}=\left\|\mathcal{E}\left(x^{*} x\right)\right\|_{p / 2}^{1 / 2} .
$$


It was shown in [20] that for every $n$ and $0<p \leq \infty$, there exists an isometric right $\mathcal{M}_{n}$-module map $u_{n, p}$ : $L_{p}^{c}\left(\mathcal{M}, \mathcal{E}_{n}\right) \rightarrow L_{p}\left(\mathcal{M}_{n} ; \ell_{2}^{c}\right)$ such that if $\left(e_{i, j}\right)_{i, j \geq 1}$ is the family of unit matrices in $B\left(\ell_{2}(\mathbb{N})\right)$, then

$$
u_{n, p}(x)^{*} u_{n, q}(y)=\mathcal{E}_{n}\left(x^{*} y\right) \otimes e_{1,1},
$$

for all $x \in L_{p}^{c}\left(\mathcal{M} ; \mathcal{E}_{n}\right)$ and $y \in L_{q}^{c}\left(\mathcal{M} ; \mathcal{E}_{n}\right)$ with $1 / p+1 / q \leq 1$. We now consider the increasing sequence of expectations $\left(\mathcal{E}_{n}\right)_{n \geq 1}$. Denote by $\mathcal{F}$ the collection of all finite sequences $\left(a_{n}\right)_{n \geq 1}$ in $L_{1}(\mathcal{M}) \cap \mathcal{M}$. For $0<p \leq \infty$, define the space $L_{p}^{\text {cond }}\left(\mathcal{M} ; \ell_{2}^{c}\right)$ to be the completion of $\mathcal{F}$ with respect to the (quasi) norm:

$$
\left\|\left(a_{n}\right)\right\|_{L_{p}^{\operatorname{cond}}\left(\mathcal{M} ; \ell_{2}^{c}\right)}=\left\|\left(\sum_{n \geq 1} \mathcal{E}_{n-1}\left|a_{n}\right|^{2}\right)^{1 / 2}\right\|_{p} .
$$

The space $L_{p}^{\text {cond }}\left(\mathcal{M} ; \ell_{2}^{c}\right)$ can be isometrically embedded into an $L_{p}$-space associated to a semifinite von Neumann algebra by means of the following map:

$$
U_{p}: L_{p}^{\text {cond }}\left(\mathcal{M} ; \ell_{2}^{c}\right) \rightarrow L_{p}\left(\mathcal{M} \bar{\otimes} B\left(\ell_{2}\left(\mathbb{N}^{2}\right)\right)\right)
$$

defined by setting

$$
U_{p}\left(\left(a_{n}\right)_{n \geq 1}\right)=\sum_{n \geq 1} u_{n-1, p}\left(a_{n}\right) \otimes e_{n, 1} .
$$

From (2.3), it follows that if $\left(a_{n}\right)_{n \geq 1} \in L_{p}^{\text {cond }}\left(\mathcal{M} ; \ell_{2}^{c}\right)$ and $\left(b_{n}\right)_{n \geq 1} \in L_{q}^{\text {cond }}\left(\mathcal{M} ; \ell_{2}^{c}\right)$ for $1 / p+1 / q \leq 1$ then

$$
U_{p}\left(\left(a_{n}\right)\right)^{*} U_{q}\left(\left(b_{n}\right)\right)=\left(\sum_{n \geq 1} \mathcal{E}_{n-1}\left(a_{n}^{*} b_{n}\right)\right) \otimes e_{1,1} \otimes e_{1,1} .
$$

In particular, $\left\|\left(a_{n}\right)\right\|_{L_{p}^{\text {cond }}\left(\mathcal{M} ; \ell_{2}^{c}\right)}=\left\|U_{p}\left(\left(a_{n}\right)\right)\right\|_{p}$ and hence $U_{p}$ is indeed an isometry. We note that $U_{p}$ is independent of $p$ in the sense of interpolation. Below, we will simply write $U$ for $U_{p}$. We refer the reader to [20] and [22] for more details on the preceding construction.

In [45], the notion of conditioned spaces were generalized to the general context of noncommutative symmetric spaces. We will only need here the special case of noncommutative Orlicz spaces. We include the details for further use.

We consider the algebraic linear map $U$ restricted to the linear space $\mathcal{F}$ that takes its values in the intersection $L_{1}\left(\mathcal{M} \bar{\otimes} B\left(\ell_{2}\left(\mathbb{N}^{2}\right)\right)\right) \cap \mathcal{M} \bar{\otimes} B\left(\ell_{2}\left(\mathbb{N}^{2}\right)\right)$. For a given sequence $\left(a_{n}\right)_{n \geq 1} \in \mathcal{F}$, we set:

$$
\left\|\left(a_{n}\right)\right\|_{L_{\Phi}^{\text {cond }}\left(\mathcal{M} ; \ell_{2}^{c}\right)}=\left\|\left(\sum_{n \geq 1} \mathcal{E}_{n-1}\left|a_{n}\right|^{2}\right)^{1 / 2}\right\|_{L_{\Phi}(\mathcal{M})}=\left\|U\left(\left(a_{n}\right)\right)\right\|_{L_{\Phi}\left(\mathcal{M} \bar{\otimes} B\left(\ell_{2}\left(\mathbb{N}^{2}\right)\right)\right)} .
$$

This is well-defined and induces a norm on the linear space $\mathcal{F}$. We define the Banach space $L_{\Phi}^{\text {cond }}\left(\mathcal{M} ; \ell_{2}^{c}\right)$ to be the completion of $\mathcal{F}$ with respect to the above norm. Then $U$ extends to an isometry from $L_{\Phi}^{\text {cond }}\left(\mathcal{M} ; \ell_{2}^{c}\right)$ into $L_{\Phi}\left(\mathcal{M} \bar{\otimes} B\left(\ell_{2}\left(\mathbb{N}^{2}\right)\right)\right)$ which we will still denote by $U$.

Similarly, we may define the corresponding row version $L_{\Phi}^{\text {cond }}\left(\mathcal{M} ; \ell_{2}^{r}\right)$ which can also be viewed as a subspace of $L_{\Phi}\left(\mathcal{M} \otimes B\left(\ell_{2}\left(\mathbb{N}^{2}\right)\right)\right)$ as row vectors.

Now we define the column/row conditioned Orlicz-Hardy spaces. Let $\mathcal{F}_{M}$ denote the set of all finite martingales in $L_{1}(\mathcal{M}) \cap \mathcal{M}$. Define $\mathrm{h}_{\Phi}^{c}(\mathcal{M})$ (respectively, $\mathrm{h}_{\Phi}^{r}(\mathcal{M})$ ) as the completion of $\mathcal{F}_{M}$ under the norm $\|x\|_{\mathrm{h}_{\Phi}^{c}}=\left\|s_{c}(x)\right\|_{L_{\Phi}(\mathcal{M})}$ (respectively, $\left.\|x\|_{\mathrm{h}_{\Phi}^{r}}=\left\|s_{r}(x)\right\|_{L_{\Phi}(\mathcal{M})}\right)$. We observe that for every $x \in \mathcal{F}_{M},\|x\|_{\mathrm{h}_{\Phi}^{c}}=\left\|\left(d x_{n}\right)\right\|_{L_{\Phi}^{\text {cond }}\left(\mathcal{M} ; \ell_{2}^{c}\right)}$. Therefore, $\mathrm{h}_{\Phi}^{c}(\mathcal{M})$ may be viewed as a subspace of $L_{\Phi}^{\text {cond }}\left(\mathcal{M} ; \ell_{2}^{c}\right)$. More precisely, we consider the map $\mathcal{D}: \mathcal{F}_{M} \rightarrow \mathcal{F}$ by setting $\mathcal{D}(x)=\left(d x_{n}\right)_{n \geq 1}$. Then $\mathcal{D}$ extends to an isometry from $\mathrm{h}_{\Phi}^{c}(\mathcal{M})$ into $L_{\Phi}^{\text {cond }}\left(\mathcal{M} ; \ell_{2}^{c}\right)$ which we will denote by $\mathcal{D}_{c}$. In the sequel, we will make frequent use of the isometric embedding:

$$
U \mathcal{D}_{c}: \mathrm{h}_{\Phi}^{c}(\mathcal{M}) \rightarrow L_{\Phi}\left(\mathcal{M} \bar{\otimes} B\left(\ell_{2}\left(\mathbb{N}^{2}\right)\right)\right)
$$


We can make similar assertions for the row case. That is, the space $\mathrm{h}_{\Phi}^{r}(\mathcal{M})$ embeds isometrically into $L_{\Phi}(\mathcal{M} \bar{\otimes}$ $B\left(\ell_{2}\left(\mathbb{N}^{2}\right)\right)$ ). We also need the diagonal Hardy space $h_{\Phi}^{d}(\mathcal{M})$ which is the space of all martingales whose martingale difference sequences belong to $L_{\Phi}\left(\mathcal{M} \bar{\otimes} \ell_{\infty}\right)$ equipped with the norm $\|x\|_{h_{\Phi}^{d}}:=\left\|\left(d x_{n}\right)\right\|_{L_{\Phi}\left(\mathcal{M} \bar{\otimes} \ell_{\infty}\right)}$. As above, we denote by $\mathcal{D}_{d}$ the isometric extension of $\mathcal{D}$ from $\mathrm{h}_{\Phi}^{d}(\mathcal{M})$ into $L_{\Phi}\left(\mathcal{M} \bar{\otimes} \ell_{\infty}\right)$. From boundedness of conditional expectations, one can easily verify that $\mathcal{D}_{d}\left(h_{\Phi}^{d}(\mathcal{M})\right)$ is a closed subspace of $L_{\Phi}\left(\mathcal{M} \bar{\otimes} \ell_{\infty}\right)$ which implies in turn that $\mathrm{h}_{\Phi}^{d}(\mathcal{M})$ is a Banach space. As noted in [45], $\mathrm{h}_{\Phi}^{d}(\mathcal{M}), \mathrm{h}_{\Phi}^{c}(\mathcal{M})$, and $\mathrm{h}_{\Phi}^{r}(\mathcal{M})$ are compatible in the sense that they embed into a larger Banach space. We now define the conditioned version of martingale Orlicz-Hardy spaces as follows. If $1 \leq p_{\Phi} \leq q_{\Phi}<2$, then

$$
\mathrm{h}_{\Phi}(\mathcal{M})=\mathrm{h}_{\Phi}^{d}(\mathcal{M})+\mathrm{h}_{\Phi}^{c}(\mathcal{M})+\mathrm{h}_{\Phi}^{r}(\mathcal{M})
$$

equipped with the norm

$$
\|x\|_{\mathrm{h}_{\Phi}}=\inf \left\{\|w\|_{\mathrm{h}_{\Phi}^{d}}+\|y\|_{\mathrm{h}_{\Phi}^{c}}+\|z\|_{\mathrm{h}_{\Phi}^{r}}\right\}
$$

where the infimum is taken over all $w \in \mathrm{h}_{\Phi}^{d}(\mathcal{M}), y \in \mathrm{h}_{\Phi}^{c}(\mathcal{M})$, and $z \in \mathrm{h}_{\Phi}^{r}(\mathcal{M})$ such that $x=w+y+z$. If $2 \leq p_{\Phi} \leq$ $q_{\Phi}<\infty$, then

$$
\mathrm{h}_{\Phi}(\mathcal{M})=\mathrm{h}_{\Phi}^{d}(\mathcal{M}) \cap \mathrm{h}_{\Phi}^{c}(\mathcal{M}) \cap \mathrm{h}_{\Phi}^{r}(\mathcal{M})
$$

equipped with the norm

$$
\|x\|_{\mathrm{h}_{\Phi}}=\max \left\{\|x\|_{\mathrm{h}_{\Phi}^{d}},\|x\|_{\mathrm{h}_{\Phi}^{c}},\|x\|_{\mathrm{h}_{\Phi}^{r}}\right\} .
$$

The reason behind the consideration of different definitions according to $q_{\Phi}<2$ or $p_{\Phi}>2$ goes back to the noncommutative Khintchine inequalities from [32,33]. For the particular case $\Phi(t)=t^{p}$ then $\mathrm{h}_{\Phi}(\mathcal{M})=\mathrm{h}_{p}(\mathcal{M})$ where $\mathrm{h}_{p}(\mathcal{M})$ is the conditioned Hardy space as defined in $[20,23]$. The space $\mathrm{h}_{\Phi}(\mathcal{M})$ is the conditioned version of martingale Orlicz Hardy spaces constructed from square functions explicitly defined in [1]. As a particular case of the extensions of the noncommutative Burkholder inequalities to general noncommutative symmetric spaces treated in [45, Theorem 3.1], we have the following identification:

$$
\mathrm{h}_{\Phi}(\mathcal{M}) \approx_{\Phi} L_{\Phi}(\mathcal{M})
$$

whenever $1<p_{\Phi} \leq q_{\Phi}<2$ or $2<p_{\Phi} \leq q_{\Phi}<\infty$.

Let us now discuss $\Phi$-moments of conditioned square functions for $x \notin L_{2}(\mathcal{M})+\mathcal{M}$. The important fact revealed by (2.5) is that if $x$ is a martingale from $\mathcal{F}_{M}$ then $s_{c}(x)$ (as defined above) can be identified to the modulus of the measurable operator $U \mathcal{D}_{c}(x)$ in the space $L_{\Phi}\left(\mathcal{M} \bar{\otimes} B\left(\ell_{2}\left(\mathbb{N}^{2}\right)\right)\right)$. We extend this identity to all martingales $x \in \mathrm{h}_{\Phi}^{c}(\mathcal{M})$. That is, for each $x \in \mathrm{h}_{\Phi}^{c}(\mathcal{M})$, we make the convention that the column conditioned square function of $x$ is given by:

$$
s_{c}(x)=\left|U \mathcal{D}_{c}(x)\right| \text {. }
$$

Similarly, we may also define the corresponding row version by setting:

$$
s_{r}(y)=s_{c}\left(y^{*}\right)=\left|U \mathcal{D}_{c}\left(y^{*}\right)\right|, \quad y \in \mathbf{h}_{\Phi}^{r}(\mathcal{M}) .
$$

Clearly, the definition of $h_{\Phi}^{c}(\mathcal{M})$ allows the identification for the norms:

$$
\left\|s_{c}(x)\right\|_{L_{\Phi}(\mathcal{M})}=\left\|U \mathcal{D}_{c}(x)\right\|_{L_{\Phi}\left(\mathcal{M} \bar{\otimes} B\left(\ell_{2}\left(\mathbb{N}^{2}\right)\right)\right)}=\|x\|_{\mathrm{h}_{\Phi}^{c}} .
$$

Accordingly, $\Phi$-moments of column conditioned square functions are then understood as:

$$
\tau\left[\Phi\left(s_{c}(x)\right)\right]=\tau \otimes \operatorname{Tr}\left[\Phi\left(\left|U \mathcal{D}_{c}(x)\right|\right)\right],
$$

where $\operatorname{Tr}$ denotes the usual trace on $B\left(\ell_{2}\left(\mathbb{N}^{2}\right)\right)$. We should warn the reader that when $x \notin L_{2}(\mathcal{M})+\mathcal{M}$, the $\Phi$-moment $\tau\left[\Phi\left(s_{c}(x)\right)\right]$ is only a suggestive notation as $s_{c}(x)$ may not exist in the sense of (2.2). We also define $\tau\left[\Phi\left(s_{r}(x)\right)\right]$ in a similar way. 
We end this subsection by recording the following simultaneous decomposition result which is the essential key ingredient to our approach. Its main feature is that one of the inequalities in the noncommutative Burkholder inequalities from [23] can be achieved with a single decomposition.

Theorem 2.5 ([45]). There exists a family $\left\{\kappa_{p}: 1<p<2\right\} \subset \mathbb{R}_{+}$satisfying the following: if $x \in L_{1}(\mathcal{M}) \cap L_{2}(\mathcal{M})$, then there exist $a \in \bigcap_{1<p<2} \mathrm{~h}_{p}^{d}(\mathcal{M}), b \in \bigcap_{1<p<2} \mathrm{~h}_{p}^{c}(\mathcal{M})$, and $c \in \bigcap_{1<p<2} \mathrm{~h}_{p}^{r}(\mathcal{M})$ such that:

(i) $x=a+b+c$;

(ii) for every $1<p<2$, the following inequality holds:

$$
\|a\|_{\mathrm{h}_{p}^{d}}+\|b\|_{\mathrm{h}_{p}^{c}}+\|c\|_{\mathrm{h}_{p}^{r}} \leq \kappa_{p}\|x\|_{p} .
$$

\section{Interpolations and some auxiliary inequalities}

In this section, we recall some basic definitions from interpolation theory and provide four inequalities that are at the core of our argument in the next section. These are stated in Proposition 3.3, Proposition 3.5, Proposition 3.6, and Proposition 3.10. Although we only need these results in the special case of various noncommutative $L_{p}$-spaces, for the sake of clarity, we chose to work with the abstract context of compatible couple of general Banach spaces. Our main references for interpolation of general Banach spaces are [4,5,27].

Let $\bar{X}=\left(X_{0}, X_{1}\right)$ be a compatible couple of Banach spaces in the sense that $X_{0}$ and $X_{1}$ are continuously embedded into a Hausdorff topological vector space. Then we can form the sum $\Sigma(\bar{X})=X_{0}+X_{1}$ and the intersection $\Delta(\bar{X})=$ $X_{0} \cap X_{1}$ which are Banach spaces under the norms

$$
\|x\|_{\Sigma(\bar{X})}=\inf \left\{\left\|x_{0}\right\|_{X_{0}}+\left\|x_{1}\right\|_{X_{1}}: x=x_{0}+x_{1}, x_{0} \in X_{0}, x_{1} \in X_{1}\right\}
$$

and

$$
\|x\|_{\Delta(\bar{X})}=\max \left\{\|x\|_{X_{0}},\|x\|_{X_{1}}\right\}
$$

respectively. A Banach space $Z$ will be called an intermediate space with respect to $\bar{X}$ if $\Delta(\bar{X}) \subseteq Z \subseteq \Sigma(\bar{X})$ with continuous embeddings. An intermediate space $Z$ is called an interpolation space if whenever a bounded linear operator $T: \Sigma(\bar{X}) \rightarrow \Sigma(\bar{X})$ is such that $T\left(X_{0}\right) \subseteq X_{0}$ and $T\left(X_{1}\right) \subseteq X_{1}$, we have $T(Z) \subseteq Z$ and

$$
\|T: Z \rightarrow Z\| \leq C \max \left\{\left\|T: X_{0} \rightarrow X_{0}\right\|,\left\|T: X_{1} \rightarrow X_{1}\right\|\right\}
$$

for some constant $C$. In this case, we write $Z \in \operatorname{Int}\left(X_{0}, X_{1}\right)$. Examples of interpolation spaces that are relevant to this article are Orlicz spaces. Indeed, we have $L_{\Phi} \in \operatorname{Int}\left(L_{p_{0}}, L_{p_{1}}\right)$ whenever $p_{0}<p_{\Phi} \leq q_{\Phi}<p_{1}$. In fact, the following noncommutative generalization of the classical Marcinkiewicz interpolation of operators was used in [1] as one of the main tools for dealing with various $\Phi$-moment inequalities. We only state here the version we need.

Theorem 3.1 ([1, Theorem 2.1]). Let $\mathcal{M}_{1}$ and $\mathcal{M}_{2}$ be two semifinite von Neumann algebras equipped with normal semifinite faithful traces $\tau_{1}$ and $\tau_{2}$, respectively. Assume that $1 \leq p_{0}<p_{1} \leq \infty$. Let $T: L_{p_{0}}\left(\mathcal{M}_{1}\right)+L_{p_{1}}\left(\mathcal{M}_{1}\right) \rightarrow$ $L_{p_{0}}\left(\mathcal{M}_{2}\right)+L_{p_{1}}\left(\mathcal{M}_{2}\right)$ be a linear operator that satisfies $T\left(L_{p_{i}}\left(\mathcal{M}_{1}\right)\right) \subseteq L_{p_{i}}\left(\mathcal{M}_{2}\right)$ for $i=0,1$. If $\Phi$ is an Orlicz function with $p_{0}<p_{\Phi} \leq q_{\Phi}<p_{1}$, then there exists a constant $C$ depending only on $p_{0}, p_{1}$, and $\Phi$ such that for every $x \in L_{\Phi}\left(\mathcal{M}_{1}\right)$,

$$
\tau_{2}[\Phi(|T x|)] \leq C \tau_{1}[\Phi(|x|)] .
$$

The following properties of conditioned Orlicz-Hardy spaces and diagonal Orlicz Hardy spaces are taken from [45, Proposition 2.8].

Lemma 3.2. Assume that $1<p_{0}<p_{\Phi} \leq q_{\Phi}<p_{1}<\infty$. Then:

(i) $\mathrm{h}_{\Phi}^{d}(\mathcal{M})$ is complemented in $L_{\Phi}\left(\mathcal{M} \bar{\otimes} \ell_{\infty}\right)$; 
(ii) $\mathrm{h}_{\Phi}^{c}(\mathcal{M})$ is complemented in $L_{\Phi}\left(\mathcal{M} \bar{\otimes} B\left(\ell_{2}\left(\mathbb{N}^{2}\right)\right)\right.$;

(iii) for $s \in\{d, c, r\}$, we have $\mathrm{h}_{\Phi}^{s}(\mathcal{M}) \in \operatorname{Int}\left(\mathrm{h}_{p_{0}}^{s}(\mathcal{M}), \mathrm{h}_{p_{1}}^{s}(\mathcal{M})\right)$.

The next proposition is the Hardy space versions of Theorem 3.1.

Proposition 3.3. Let $\mathcal{N}$ be a semifinite von Neumann algebra equipped with a normal semifinite faithful trace $\sigma$. Assume that $1<p_{0}<p_{1}<\infty$. Let $s \in\{d, c\}$ and $T: \mathrm{h}_{p_{0}}^{s}(\mathcal{M})+\mathrm{h}_{p_{1}}^{s}(\mathcal{M}) \rightarrow L_{p_{0}}(\mathcal{N})+L_{p_{1}}(\mathcal{N})$ be a linear operator that satisfies $T\left(\mathrm{~h}_{p_{i}}^{s}(\mathcal{M})\right) \subseteq L_{p_{i}}(\mathcal{N})$ for $i=0$, 1. If $\Phi$ is an Orlicz function with $p_{0}<p_{\Phi} \leq q_{\Phi}<p_{1}$, then there exists a constant $C$ depending only on $p_{0}, p_{1}$, and $\Phi$ such that:

(i) If $s=d$ and $x \in \mathrm{h}_{\Phi}^{d}(\mathcal{M})$, then $\sigma[\Phi(|T x|)] \leq C \sum_{n>1} \tau\left[\Phi\left(\left|d x_{n}\right|\right)\right]$.

(ii) If $s=c$ and $y \in \mathrm{h}_{\Phi}^{c}(\mathcal{M})$, then $\sigma[\Phi(|T y|)] \leq C \tau\left[\Phi\left(s_{c}(y)\right)\right]$.

Similarly, if $S: L_{p_{0}}(\mathcal{N})+L_{p_{1}}(\mathcal{N}) \rightarrow \mathrm{h}_{p_{0}}^{s}(\mathcal{M})+\mathrm{h}_{p_{1}}^{s}(\mathcal{M})$ is a linear operator that satisfies $S\left(L_{p_{i}}(\mathcal{N})\right) \subseteq \mathrm{h}_{p_{i}}^{s}(\mathcal{M})$ for $i=0,1$, then there exists a constant $C$ depending only on $p_{0}, p_{1}$, and $\Phi$ such that:

(iii) If $s=d$ and $x \in L_{\Phi}(\mathcal{N})$, then $\sum_{n \geq 1} \tau\left[\Phi\left(\left|d_{n}(S x)\right|\right)\right] \leq C \sigma[\Phi(|x|)]$ where $\left(d_{n}(S x)\right)_{n \geq 1}$ denotes the martingale difference sequence of the martingale associated with $S x$.

(iv) If $s=c$ and $y \in L_{\Phi}(\mathcal{N})$, then $\tau\left[\Phi\left(s_{c}(S y)\right)\right] \leq C \sigma[\Phi(|y|)]$.

Proof. We begin with the diagonal part. Let $\Theta: L_{p_{0}}\left(\mathcal{M} \bar{\otimes} \ell_{\infty}\right)+L_{p_{1}}\left(\mathcal{M} \bar{\otimes} \ell_{\infty}\right) \rightarrow \mathrm{h}_{p_{0}}^{d}(\mathcal{M})+\mathrm{h}_{p_{1}}^{d}(\mathcal{M})$ be the bounded projection defined by: $\Theta\left(\left(a_{n}\right)_{n \geq 1}\right)=\sum_{n \geq 1} \mathcal{E}_{n}\left(a_{n}\right)-\mathcal{E}_{n-1}\left(a_{n}\right)$. It is clear that $T \Theta\left[L_{p_{i}}\left(\mathcal{M} \bar{\otimes} \ell_{\infty}\right)\right] \subset L_{p_{i}}(\mathcal{N})$ for $i=0,1$. It follows from Theorem 3.1 that $T \Theta\left[L_{\Phi}\left(\mathcal{M} \bar{\otimes} \ell_{\infty}\right)\right] \subset L_{\Phi}(\mathcal{N})$ and there exists a constant $C=C\left(p_{0}, p_{1}, \Phi\right)$ such that:

$$
\sigma\left[\Phi\left(\left|T \Theta\left(\left(a_{n}\right)_{n}\right)\right|\right)\right] \leq C \tau \otimes \gamma\left[\Phi\left(\left|\left(a_{n}\right)_{n}\right|\right)\right],
$$

where $\tau \otimes \gamma$ is the natural trace of $\mathcal{M} \bar{\otimes} \ell_{\infty}$. Let $x \in \mathrm{h}_{\Phi}^{d}(\mathcal{M})$. When applied to the operator $\mathcal{D}_{d}(x) \in L_{\Phi}\left(\mathcal{M} \bar{\otimes} \ell_{\infty}\right)$, the above inequality yields the desired inequality.

Now, we verify the column case. Let $\mathcal{S}=\mathcal{M} \otimes B\left(\ell_{2}\left(\mathbb{N}^{2}\right)\right)$ equipped with its natural trace $\tau \otimes \operatorname{Tr}$. Define $\Pi$ : $L_{p_{0}}(\mathcal{S})+L_{p_{1}}(\mathcal{S}) \rightarrow \mathrm{h}_{p_{0}}^{c}(\mathcal{M})+\mathrm{h}_{p_{1}}^{c}(\mathcal{M})$ be the projection guaranteed by Lemma 3.2. Then we have, $T \Pi\left[L_{p_{i}}(\mathcal{S})\right] \subset$ $L_{p_{i}}(\mathcal{N})$ for $i=0,1$. As above, we deduce from Theorem 3.1 that $T \Pi\left[L_{\Phi}(\mathcal{S})\right] \subset L_{\Phi}(\mathcal{N})$ and there exists a constant $C=C_{p_{0}, p_{1}, \Phi}$ such that for every $a \in L_{\Phi}(\mathcal{S})$,

$$
\sigma[\Phi(|T \Pi(a)|)] \leq C \tau \otimes \operatorname{Tr}[\Phi(|a|)] .
$$

Let $y \in \mathrm{h}_{\Phi}^{c}(\mathcal{M})$ and take $a=U \mathcal{D}_{c}(y)$. For this special case, the preceding inequality clearly translates into the inequality in item (ii).

Items (iii) and (iv) follow from composing $S$ with the isometric embeddings $\mathcal{D}_{d}: \mathrm{h}_{p_{i}}^{d}(\mathcal{M}) \rightarrow L_{p_{i}}\left(\mathcal{M} \bar{\otimes} \ell_{\infty}\right)$ and $U \mathcal{D}_{c}: h_{p_{i}}^{c}(\mathcal{M}) \rightarrow L_{p_{i}}(\mathcal{S})$ for $i=0,1$.

We now turn our attention to specific types of interpolations. A fundamental notion for real interpolation theory is the $K$-functional. This is given by setting:

$$
K(t, x)=K(t, x ; \bar{X})=\inf \left\{\left\|x_{0}\right\|_{X_{0}}+t\left\|x_{1}\right\|_{X_{1}}: x=x_{0}+x_{1}\right\}, \quad x \in \Sigma(\bar{X}) .
$$

We will also need a dual notion known as the $J$-functional defined by

$$
J(t, x)=J(t, x ; \bar{X})=\max \left\{\|x\|_{X_{0}}, t\|x\|_{X_{1}}\right\}, \quad x \in \Delta(\bar{X}) .
$$

These two notions will be heavily used in the sequel.

We recall that by a representation of $x \in \Sigma(\bar{X})$ with respect to the couple $\bar{X}$, we mean a measurable function $u:(0, \infty) \rightarrow \Delta(\bar{X})$ satisfying

$$
x=\int_{0}^{\infty} u(t) \frac{d t}{t},
$$


where the convergence of the integral is taken in $\Sigma(\bar{X})$. Similarly, a discrete representation of $x$ with respect to the couple $\bar{X}$ is a series

$$
x=\sum_{\nu \in \mathbb{Z}} u_{\nu}
$$

with $u_{v} \in \Delta(\bar{X})$ for all $v \in \mathbb{Z}$ and the convergence of the series taken in the Banach space $\Sigma(\bar{X})$.

Definition 3.4. Given a compatible couple $\bar{X}$ and $0 \leq \theta \leq 1$, we say that an intermediate space $Z$ of $\bar{X}$ belongs to

(i) the class $\mathcal{C}_{K}(\theta, \bar{X})$ if there exists a constant $C_{1}$ such that for every $x \in Z$ and $t>0$, the following holds:

$$
K(t, x) \leq C_{1} t^{\theta}\|x\|_{Z} .
$$

(ii) the class $\mathcal{C}_{J}(\theta, \bar{X})$ if there exists a constant $C_{2}$ such that for every $x \in \Delta(\bar{X})$ and $t>0$, the following holds:

$$
\|x\|_{Z} \leq C_{2} t^{-\theta} J(t, x) \text {. }
$$

Examples of spaces belonging to the class $\mathcal{C}_{K}(\theta, \bar{X})$ are those real interpolation spaces constructed using the $K$-method. Namely, the spaces $\left(X_{0}, X_{1}\right)_{\theta, p, K}$ (we refer to [5] for the definition of $\left.\|\cdot\|_{\theta, p, K}\right)$. The corresponding statement is also valid for the class $\mathcal{C}_{J}(\theta, \bar{X})$. That is, $\left(X_{0}, X_{1}\right)_{\theta, p, J}$ belongs to $\mathcal{C}_{J}(\theta, \bar{X})$. In particular, for $\theta=1-p^{-1}$, $L_{p}$ belongs to both $\mathcal{C}_{K}(\theta)$ and $\mathcal{C}_{J}(\theta)$ for the couple $\left(L_{1}, L_{\infty}\right)$. A noncommutative analogue of the latter statement will be used in the sequel.

The next two propositions deal with reiteration type inequalities involving convex functions.

Proposition 3.5. Let $\bar{X}=\left(X_{0}, X_{1}\right)$ and $\bar{Y}=\left(Y_{0}, Y_{1}\right)$ be compatible couples of Banach spaces and $0 \leq \theta_{0}<\theta_{1} \leq 1$. Assume that $Y_{i}$ belongs to the class $\mathcal{C}_{K}\left(\theta_{i}, \bar{X}\right)$ for $i=0,1$. Then the following inequality holds:

$$
\int_{0}^{\infty} \Phi\left[t^{-1} K(t, y ; \bar{X})\right] d t \lesssim_{\Phi, \theta_{0}, \theta_{1}} \int_{0}^{\infty} \Phi\left[t^{-1+\theta_{0}} K\left(t^{\theta_{1}-\theta_{0}}, y ; \bar{Y}\right)\right] d t, \quad y \in \Sigma(\bar{Y}) .
$$

Proof. From the assumptions, there exist constants $C_{0}$ and $C_{1}$ such that if $y=y_{0}+y_{1} \in \Sigma(\bar{Y})$ then for every $t>0$,

$$
K\left(t, y_{0} ; \bar{X}\right) \leq C_{0} t^{\theta_{0}}\left\|y_{0}\right\|_{Y_{0}} \quad \text { and } \quad K\left(t, y_{1} ; \bar{X}\right) \leq C_{1} t^{\theta_{1}}\left\|y_{1}\right\|_{Y_{1}} .
$$

It follows that $K(t, y ; \bar{X}) \leq C_{0} t^{\theta_{0}}\left\|y_{0}\right\|_{Y_{0}}+C_{1} t^{\theta_{1}}\left\|y_{1}\right\|_{Y_{1}}$. Taking the infimum over all such decompositions of $y$, we have for $C=\max \left\{C_{0}, C_{1}\right\}$ that

$$
K(t, y ; \bar{X}) \leq C t^{\theta_{0}} K\left(t^{\theta_{1}-\theta_{0}}, y ; \bar{Y}\right) .
$$

Since $\Phi$ is increasing and satisfies the $\Delta_{2}$-condition, we may conclude that

$$
\begin{aligned}
\int_{0}^{\infty} \Phi\left[t^{-1} K(t, y ; \bar{X})\right] d t & \leq \int_{0}^{\infty} \Phi\left[C t^{-1+\theta_{0}} K\left(t^{\theta_{1}-\theta_{0}}, y ; \bar{Y}\right)\right] d t \\
& \lesssim \int_{0}^{\infty} \Phi\left[t^{-1+\theta_{0}} K\left(t^{\theta_{1}-\theta_{0}}, y ; \bar{Y}\right)\right] d t
\end{aligned}
$$

The fact that the constant depends only on $\Phi, \theta_{0}$, and $\theta_{1}$ is clear from the argument.

A dual version of the preceding proposition reads as follows:

Proposition 3.6. Let $\bar{X}=\left(X_{0}, X_{1}\right)$ and $\bar{Y}=\left(Y_{0}, Y_{1}\right)$ be compatible couples of Banach spaces and $0 \leq \theta_{0}<\theta_{1} \leq 1$. Assume that $Y_{i}$ belongs to the class $\mathcal{C}_{J}\left(\theta_{i}, \bar{X}\right)$ for $i=0,1$. Let $y \in \Delta(\bar{Y})$ and $u(\cdot)$ be a representation of $y$ for the couple $\bar{X}$. If $u(\cdot)$ is also a representation of $x$ for the couple $\bar{Y}$ then the following inequality holds:

$$
\int_{0}^{\infty} \Phi\left[t^{-1+\theta_{0}} J\left(t^{\theta_{1}-\theta_{0}}, u(t) ; \bar{Y}\right)\right] d t \lesssim \Phi, \theta_{0}, \theta_{1} \int_{0}^{\infty} \Phi\left[t^{-1} J(t, u(t) ; \bar{X})\right] d t .
$$


Proof. The argument is nearly identical to the one used earlier. We include the details for completeness. For the inequality, we have from the assumptions that there exist constants $C_{0}$ and $C_{1}$ such that for every $t>0$,

$$
\|u(t)\|_{Y_{0}} \leq C_{0} t^{-\theta_{0}} J(t, u(t) ; \bar{X}) \quad \text { and } \quad\|u(t)\|_{Y_{1}} \leq C_{1} t^{-\theta_{1}} J(t, u(t) ; \bar{X})
$$

The latter is equivalent to the inequality

$$
t^{\theta_{1}-\theta_{0}}\|u(t)\|_{Y_{1}} \leq C_{1} t^{-\theta_{0}} J(t, u(t) ; \bar{X})
$$

This implies that for $C=\max \left\{C_{0}, C_{1}\right\}$, we have $J\left(t^{\theta_{1}-\theta_{0}}, u(t), \bar{Y}\right) \leq C t^{-\theta_{0}} J(t, u(t) ; \bar{X})$. That is,

$$
t^{-1+\theta_{0}} J\left(t^{\theta_{1}-\theta_{0}}, u(t) ; \bar{Y}\right) \leq C t^{-1} J(t, u(t) ; \bar{X}) .
$$

Since $\Phi$ satisfies the $\Delta_{2}$-condition, we conclude as before that

$$
\begin{aligned}
\int_{0}^{\infty} \Phi\left[t^{-1+\theta_{0}} J\left(t^{\theta_{1}-\theta_{0}}, u(t) ; \bar{Y}\right)\right] d t & \leq \int_{0}^{\infty} \Phi\left[C t^{-1} J(t, u(t) ; \bar{X})\right] d t \\
& \lesssim \int_{0}^{\infty} \Phi\left[t^{-1} J(t, u(t) ; \bar{X})\right] d t
\end{aligned}
$$

As noted in the previous proposition, the constant involved depends only on $\Phi, \theta_{0}$, and $\theta_{1}$.

In preparation for the next proposition, let us review some basic facts about the following classical operators. For $f \in L_{0}(0, \infty)$, we define the Calderón's operators by setting for $1 \leq p<q<\infty$,

$$
S_{p, q} f(t)=t^{-\frac{1}{p}} \int_{0}^{t} s^{\frac{1}{p}} f(s) \frac{d s}{s}+t^{-\frac{1}{q}} \int_{t}^{\infty} s^{\frac{1}{q}} f(s) \frac{d s}{s}, \quad t>0
$$

and for $1 \leq p<\infty$,

$$
S_{p, \infty} f(t)=t^{-\frac{1}{p}} \int_{0}^{t} s^{\frac{1}{p}} f(s) \frac{d s}{s}, \quad t>0 .
$$

Connections between Calderón operators and interpolation theory are well-established in the literature. It was noted in [4, Proposition 5.5] that for $1 \leq p<q \leq \infty$, the linear operator $S_{p, q}$ is simultaneously of weak-types $(p, p)$ and $(q, q)$. Thus, by standard use of Marcinkiewicz interpolation, we have the following well-known properties:

\section{Lemma 3.7.}

(i) For every $1 \leq p<r<q, S_{p, q}$ is a bounded linear operator on $L_{r}(0, \infty)$;

(ii) for $1 \leq p<r \leq \infty, S_{p, \infty}$ is a bounded linear operator on $L_{r}(0, \infty)$.

As immediate consequences, we also have the following $\Phi$-moment versions:

Lemma 3.8. If $1 \leq p<p_{\Phi}<q_{\Phi}<q<\infty$, then for every $f \in L_{\Phi}(0, \infty)$,

$$
\int_{0}^{\infty} \Phi\left[\left|S_{p, \infty} f(t)\right|\right] d t \lesssim_{\Phi, p} \int_{0}^{\infty} \Phi[|f(t)|] d t
$$

and

$$
\int_{0}^{\infty} \Phi\left[\left|S_{p, q} f(t)\right|\right] d t \lesssim_{\Phi, p, q} \int_{0}^{\infty} \Phi[|f(t)|] d t .
$$


Proof. From Lemma 3.7, both $S_{p, \infty}$ and $S_{p, q}$ are bounded simultaneously on $L_{r_{1}}(0, \infty)$ and $L_{r_{2}}(0, \infty)$ whenever $p<r_{1}<p_{\Phi} \leq q_{\Phi}<r_{2}<q$. The two inequalities as stated follow immediately from applying Theorem 3.1 to the abelian von Neumann algebra $L_{\infty}(0, \infty)$.

The next result is a weighted version of the previous lemma. We only consider the special case that we will use.

Lemma 3.9. Let $1<p<p_{\Phi} \leq q_{\Phi}<q<\infty$. If $g$ is a nonnegative decreasing function defined in $(0, \infty)$ with $t \mapsto t^{-1 / p} g\left(t^{1 / p-1 / q}\right)$ belongs to $L_{\Phi}(0, \infty)$, then

$$
\int_{0}^{\infty} \Phi\left[t^{-1 / q} S_{1, \infty} g\left(t^{1 / p-1 / q}\right)\right] d t \simeq \Phi, p, q \int_{0}^{\infty} \Phi\left[t^{-1 / q} g\left(t^{1 / p-1 / q}\right)\right] d t
$$

Proof. Since $g \leq S_{1, \infty} g$, one inequality is immediate. For the non trivial inequality, let $\theta=1 / p-1 / q$ and define the function

$$
\psi(t)=t^{-1 / q} S_{1, \infty} g\left(t^{\theta}\right)=t^{-1 / p} \int_{0}^{t^{\theta}} g(s) d s, \quad t>0 .
$$

Using the substitution $s=w^{\theta}$, we have

$$
\begin{aligned}
\psi(t) & =\theta t^{-1 / p} \int_{0}^{t} g\left(w^{\theta}\right) w^{\theta-1} d w \\
& =\theta t^{-1 / p} \int_{0}^{t} w^{1 / p} w^{-1 / q} g\left(w^{\theta}\right) \frac{d w}{w} \\
& =\theta S_{p, \infty}\left(h_{\theta}\right)(t),
\end{aligned}
$$

where $h_{\theta}$ is the function $t \mapsto t^{-1 / q} g\left(t^{\theta}\right)$. We may deduce that

$$
\begin{aligned}
\int_{0}^{\infty} \Phi\left[t^{-1 / q} S_{1, \infty} g\left(t^{\theta}\right)\right] d t & \leq \int_{0}^{\infty} \Phi\left[\theta S_{p, \infty}\left(h_{\theta}\right)(t)\right] d t \\
& \leq \int_{0}^{\infty} \Phi\left[S_{p, \infty}\left(h_{\theta}\right)(t)\right] d t \\
& \lesssim \int_{0}^{\infty} \Phi\left[h_{\theta}(t)\right] d t
\end{aligned}
$$

where the last inequality comes from the first inequality in Lemma 3.8. This is the desired inequality.

We now state the following weighted comparison between $K$-functionals and $J$-functionals.

Proposition 3.10. Assume that $1<p<p_{\Phi} \leq q_{\Phi}<q<\infty$ and $\bar{Y}$ is an interpolation couple. Then for every $y \in$ $\Sigma(\bar{Y})$,

$$
\int_{0}^{\infty} \Phi\left[t^{-1 / p} K\left(t^{1 / p-1 / q}, y ; \bar{Y}\right)\right] d t \lesssim_{\Phi, p, q} \inf \left\{\int_{0}^{\infty} \Phi\left[t^{-1 / p} J\left(t^{1 / p-1 / q}, u\left(t^{1 / p-1 / q}\right) ; \bar{Y}\right)\right] d t\right\},
$$

where the infimum is taken over all representations $u(\cdot)$ of $y$.

Proof. We will deduce the inequality in two steps. First, we recall the notion of $j$-functionals related to the interpolation couple $\bar{Y}$. Suppose that $y \in \Sigma(\bar{Y})$ admits a representation $u(\cdot)$. We define

$$
j(s, u)=j(s, u ; \bar{Y})=\int_{s}^{\infty} t^{-1} J(t, u(t)) d t / t, \quad s>0 .
$$


We will verify first that the inequality stated in the proposition holds for the $j$-functional in place of the $J$-functional. That is, we claim that

$$
\int_{0}^{\infty} \Phi\left[t^{-1 / p} K\left(t^{1 / p-1 / q}, y\right)\right] d t \lesssim \inf \left\{\int_{0}^{\infty} \Phi\left[t^{-1 / q} j\left(t^{1 / p-1 / q}, u\right)\right] d t\right\},
$$

where the infimum is taken over all representations $u(\cdot)$ of $y$ in the couple $\bar{Y}$.

To prove this assertion, we fix a representation $u(\cdot)$ of $y$. As above, we let $\theta=1 / p-1 / q$. The crucial point of the argument is given by the following inequality:

$$
K\left(t^{\theta}, y\right) \leq \int_{0}^{t^{\theta}} j(s, u) d s, \quad t>0 .
$$

A verification of this fact can be found for instance in [3, p. 427]. Since $t^{-1 / p}=t^{-1 / q} t^{-\theta}$, the preceding inequality can be rewritten in the following form:

$$
t^{-1 / p} K\left(t^{\theta}, y\right) \leq t^{-1 / q} S_{1, \infty}(j(\cdot, u))\left(t^{\theta}\right), \quad t>0 .
$$

Since $j(\cdot, u)$ is a decreasing function, after applying the function $\Phi$ on both sides of the preceding inequality and taking integrals, (3.1) follows immediately from Lemma 3.9.

Next, we will verify that for any representation $u(\cdot)$ of $y$, we have

$$
\int_{0}^{\infty} \Phi\left[t^{-1 / q} j\left(t^{1 / p-1 / q}, u\right)\right] d t \lesssim \int_{0}^{\infty} \Phi\left[t^{-1 / p} J\left(t^{1 / p-1 / q}, u\left(t^{1 / p-1 / q}\right)\right)\right] d t
$$

Indeed, from the definition of $j(\cdot, u)$, we have $j\left(t^{\theta}, u\right)=\int_{t^{\theta}}^{\infty} s^{-1} J(s, u(s)) d s / s$. Therefore, for every $t>0$,

$$
t^{-1 / q} j\left(t^{\theta}, u\right)=t^{-1 / q} \int_{t^{\theta}}^{\infty} s^{-1} J(s, u(s)) d s / s .
$$

Using the substitution $s=w^{\theta}$, the preceding equality gives for every $t>0$,

$$
\begin{aligned}
t^{-1 / q} j\left(t^{\theta}, u\right) & =\theta t^{-1 / q} \int_{t}^{\infty} J\left(w^{\theta}, u\left(w^{\theta}\right)\right) w^{-2 \theta} w^{\theta-1} d w \\
& \leq t^{-1 / q} \int_{t}^{\infty} J\left(w^{\theta}, u\left(w^{\theta}\right)\right) w^{-\theta} d w / w \\
& \leq t^{-1 / q} \int_{t}^{\infty} w^{1 / q} w^{-1 / p} J\left(w^{\theta}, u\left(w^{\theta}\right)\right) d w / w \\
& \leq S_{p, q}\left(\psi_{\theta}\right)(t)
\end{aligned}
$$

where $\psi_{\theta}(t)=t^{-1 / p} J\left(t^{\theta}, u\left(t^{\theta}\right)\right)$. We deduce that

$$
\int_{0}^{\infty} \Phi\left[t^{-1 / q} j\left(t^{\theta}, u\right)\right] d t \leq \int_{0}^{\infty} \Phi\left[S_{p, q}\left(\psi_{\theta}\right)(t)\right] d t \lesssim \int_{0}^{\infty} \Phi\left[\psi_{\theta}(t)\right] d t
$$

where the second inequality comes from the second inequality in Lemma 3.8. This is the desired inequality. Combining (3.1) and (3.2) clearly gives the proposition.

Remark 3.11. By choosing a representation $u(\cdot)$ satisfying $J(t, u(t)) \leq C K(t, y)$ (for some absolute constant $C$ ), the converse of the inequality stated in Proposition 3.10 clearly holds but this fact will not be needed.

We conclude this section with a discretization of the second integral appearing in Proposition 3.10. 
Lemma 3.12. Let $1<p<q<\infty$ and $\operatorname{set} \theta=1 / p-1 / q$. Fix $y \in \Sigma(\bar{Y})$.

(i) Assume that $y=\int_{0}^{\infty} u(t) d t / t$ is a representation of $y$. If for every $v \in \mathbb{Z}$, we set $u_{v}=\int_{2^{v}}^{2^{v+1}} u(t) d t / t$, then $y=\sum_{v \in \mathbb{Z}} u_{v}$ is a discrete representation of $y$ and

$$
\sum_{v \in \mathbb{Z}} 2^{v / \theta} \Phi\left[2^{-v /(\theta p)} J\left(2^{v}, u_{v} ; \bar{Y}\right)\right] \lesssim_{\Phi, p, q} \int_{0}^{\infty} \Phi\left[t^{-1 / p} J\left(t^{1 / p-1 / q}, u\left(t^{1 / p-1 / q}\right) ; \bar{Y}\right)\right] d t .
$$

(ii) Conversely, assume that $y$ admits a discrete representation $y=\sum_{v \in \mathbb{Z}} u_{v}$. If we set for $t \in\left[2^{v}, 2^{v+1}\right), u(t)=$ $u_{v} /(\log 2)$ then $y=\int_{0}^{\infty} u(t) d t / t$ is a representation of $y$ and

$$
\int_{0}^{\infty} \Phi\left[t^{-1 / p} J\left(t^{1 / p-1 / q}, u\left(t^{1 / p-1 / q}\right) ; \bar{Y}\right)\right] d t \lesssim_{\Phi, p, q} \sum_{v \in \mathbb{Z}} 2^{v / \theta} \Phi\left[2^{-v /(\theta p)} J\left(2^{v}, u_{v} ; \bar{Y}\right)\right] .
$$

Sketch of the proof. Fix a representation $u(\cdot)$ of $y$. A simple use of substitution gives,

$$
\int_{0}^{\infty} \Phi\left[t^{-1 / p} J\left(t^{1 / p-1 / q}, u\left(t^{1 / p-1 / q}\right)\right)\right] d t=\theta^{-1} \int_{0}^{\infty} \Phi\left[t^{-1 /(\theta p)} J(t, u(t))\right] t^{\theta^{-1}} d t / t .
$$

Using the integral in the right hand side of the above equality, the verification of the two inequalities in the lemma is a simple adaptation of standard arguments from interpolation theory which we leave for the reader.

\section{4. $\Phi$-Moment versions of Burkholder inequalities}

In this section, we present our primary objective. That is, to formulate $\Phi$-moment extensions of the noncommutative Burkholder inequalities. The following theorem is the main result of this paper. It extends the noncommutative Burkholder inequalities (for the case $1<p<2$ ) from [23, Theorem 6.1] to moment inequalities involving Orlicz functions.

Theorem 4.1. Let $\Phi$ be an Orlicz function satisfying $1<p_{\Phi} \leq q_{\Phi}<2$. There exist positive constants $\delta_{\Phi}$ and $\eta_{\Phi}$ depending only on $\Phi$ such that for every martingale $x \in L_{\Phi}(\mathcal{M})$, the following inequalities hold:

$$
\delta_{\Phi}^{-1} S_{\Phi}(x) \leq \tau[\Phi(|x|)] \leq \eta_{\Phi} S_{\Phi}(x),
$$

where $S_{\Phi}(x)=\inf \left\{\tau\left[\Phi\left(s_{c}\left(x^{c}\right)\right)\right]+\tau\left[\Phi\left(s_{r}\left(x^{r}\right)\right)\right]+\sum_{n \geq 1} \tau\left[\Phi\left(\left|d x_{n}^{d}\right|\right)\right]\right\}$ with the infimum being taken over all $x^{c} \in$ $\mathrm{h}_{\Phi}^{c}(\mathcal{M}), x^{r} \in \mathrm{h}_{\Phi}^{r}(\mathcal{M})$, and $x^{d} \in \mathrm{h}_{\Phi}^{d}(\mathcal{M})$ such that $x=x^{c}+x^{r}+x^{d}$.

Throughout the proof, we fix $p$ and $q$ such that $1<p<p_{\Phi} \leq q_{\Phi}<q<2$. First, we prove the second inequality of $\left(B_{\Phi}\right)$. This will be deduced from interpolating the noncommutative Burkholder inequalities. Indeed, since $1<$ $p, q<2$, the noncommutative Burkholder inequalities implies that for $s \in\{d, c, r\}, \mathrm{h}_{p}^{s}(\mathcal{M}) \subset L_{p}(\mathcal{M})$ and $\mathrm{h}_{q}^{s}(\mathcal{M}) \subset$ $L_{q}(\mathcal{M})$. By Proposition 3.3, it follows that for every $y \in \mathrm{h}_{\Phi}^{d}(\mathcal{M})$, we have

$$
\tau[\Phi(|y|)] \leq C_{\Phi} \sum_{n \geq 1} \tau\left[\Phi\left(\left|d y_{n}\right|\right)\right] .
$$

Similarly, for $z \in \mathrm{h}_{\Phi}^{c}(\mathcal{M})$, we have

$$
\tau[\Phi(|z|)] \leq C_{\Phi}^{\prime} \tau\left[\Phi\left(s_{c}(z)\right)\right] .
$$

Considering adjoint operators, we may also state that for $w \in \mathrm{h}_{\Phi}^{r}(\mathcal{M})$, we have

$$
\tau[\Phi(|w|)] \leq C_{\Phi}^{\prime} \tau\left[\Phi\left(s_{r}(w)\right)\right]
$$


Now, let $x=x^{c}+x^{r}+x^{d}$ with $x^{c} \in \mathrm{h}_{\Phi}^{c}(\mathcal{M}), x^{r} \in \mathrm{h}_{\Phi}^{r}(\mathcal{M})$, and $x^{d} \in \mathrm{h}_{\Phi}^{d}(\mathcal{M})$. We deduce from (4.2), (4.3), and (4.1) that

$$
\begin{aligned}
\tau[\Phi(|x|)] & \leq C_{\Phi}^{\prime \prime}\left\{\tau\left[\Phi\left(\left|x^{d}\right|\right)\right]+\tau\left[\Phi\left(\left|x^{c}\right|\right)\right]+\tau\left[\Phi\left(\left|x^{r}\right|\right)\right]\right\} \\
& \leq C_{\Phi}^{\prime \prime} \max \left\{C_{\Phi}, C_{\Phi}^{\prime}\right\}\left\{\tau\left[\Phi\left(s_{c}\left(x^{c}\right)\right)\right]+\tau\left[\Phi\left(s_{r}\left(x^{r}\right)\right)\right]+\sum_{n \geq 1} \tau\left[\Phi\left(\left|d x_{n}^{d}\right|\right)\right]\right\} .
\end{aligned}
$$

Taking the infimum over all such decompositions completes the proof of the second inequality of $\left(B_{\Phi}\right)$.

Now, we proceed with the proof of the first inequality of $\left(B_{\Phi}\right)$. The proof will be done in several steps and rests upon the fact noted earlier that the Orlicz space $L_{\Phi}(\mathcal{M})$ is an interpolation space for the compatible couple $\left(L_{p}(\mathcal{M}), L_{q}(\mathcal{M})\right)$. A fortiori, it is an interpolation space for the compatible couple $\left(L_{1}(\mathcal{M}), \mathcal{M}\right)$. Our approach was motivated by the following formula on $K$-functionals: for $x \in L_{1}(\mathcal{M})+\mathcal{M}$,

$$
K\left(t, x ; L_{1}(\mathcal{M}), \mathcal{M}\right)=\int_{0}^{t} \mu_{s}(x) d s, \quad t>0 .
$$

This fact can be found for instance in [41, Corollary 2.3]. We make the following crucial observation:

$$
\tau[\Phi(|x|)]=\int_{0}^{\infty} \Phi\left(\mu_{t}(x)\right) d t \simeq \int_{0}^{\infty} \Phi\left[t^{-1} K(t, x)\right] d t
$$

where the equivalence is taken from the property of Calderón's operator stated in Lemma 3.8. Thus, proving the first inequality in $\left(B_{\Phi}\right)$ amounts to finding suitable estimate for the integral of the function $t \mapsto \Phi\left[t^{-1} K(t, x)\right]$ from below. However, as it will be clear from the steps taken below, the $J$-functionals computed with respect to the compatible couple $\left(L_{p}(\mathcal{M}), L_{q}(\mathcal{M})\right)$ turn out to be the right framework for this stated goal. Below, $C_{\Phi, p, q}$ denotes a positive constant whose value may change from one line to the next.

- We assume first that $x \in L_{1}(\mathcal{M}) \cap \mathcal{M}$.

Step 1. Choose a representation $u(\cdot)$ of $x$ in the compatible couple $\left(L_{1}(\mathcal{M}), \mathcal{M}\right)$ such that:

$$
J(t, u(t)) \leq C K(t, x), \quad t>0,
$$

where $C$ is an absolute constant. Thus, since $\Phi$ has the $\Delta_{2}$-condition, we have from (4.4) and (4.5) that

$$
\int_{0}^{\infty} \Phi\left[t^{-1} J(t, u(t))\right] d t \leq C_{\Phi} \tau[\Phi(|x|)] .
$$

Step 2. Changing into the compatible couple $\left(L_{p}(\mathcal{M}), L_{q}(\mathcal{M})\right)$. This is done through Proposition 3.6. Denote by $\bar{X}$ the compatible couple $\left(L_{1}(\mathcal{M}), \mathcal{M}\right)$. If we set $\theta_{0}=1-p^{-1}$ and $\theta_{1}=1-q^{-1}$ then $L_{p}(\mathcal{M})$ and $L_{q}(\mathcal{M})$ belong to the classes $\mathcal{C}_{J}\left(\theta_{0}, \bar{X}\right)$ and $\mathcal{C}_{J}\left(\theta_{1}, \bar{X}\right)$, respectively. We claim that $u(\cdot)$ is also a representation of $x$ for the compatible couple $\left(L_{p}(\mathcal{M}), L_{q}(\mathcal{M})\right)$. To verify this claim, fix $p<r<q$. Since $x \in L_{1}(\mathcal{M}) \cap \mathcal{M}$, it belongs to $L_{r}(\mathcal{M})$. Let $\theta=1-r^{-1}$. We recall that $L_{r}(\mathcal{M})=\left(L_{1}(\mathcal{M}), \mathcal{M}\right)_{\theta, r, K}$ (with equivalent norms) where $(\cdot, \cdot)_{\theta, r, K}$ denotes the real interpolation using the $K$-method (see [5]). We have by the definition of $\left(L_{1}(\mathcal{M}), \mathcal{M}\right)_{\theta, r, K}$ that the function $t^{-\theta} K(t, x ; \bar{X}) \in L_{r}\left(\mathbb{R}_{+}, d t / t\right)$. From (4.5), we also have $t^{-\theta} J(t, u(t) ; \bar{X}) \in L_{r}\left(\mathbb{R}_{+}, d t / t\right)$. It is implicit in the proof of [9, Proposition 3.3.19, pp. 177-178] that the latter assertion implies that the integral $\int_{0}^{\infty} u(t) d t / t$ is convergent in $L_{p}(\mathcal{M})+L_{q}(\mathcal{M})$. This verifies the claim.

With the above observations, it is clear that Proposition 3.6 applies to our situation. We should point out here that the only reason for considering $x \in L_{1}(\mathcal{M}) \cap \mathcal{M}$ is to insure that $u(\cdot)$ is a representation of $x$ for both compatible couples.

Putting (4.6) together with Proposition 3.6 yields:

$$
\int_{0}^{\infty} \Phi\left[t^{-1 / p} J\left(t^{1 / p-1 / q}, u(t) ; L_{p}(\mathcal{M}), L_{q}(\mathcal{M})\right)\right] d t \leq C_{\Phi, p, q} \tau[\Phi(|x|)] .
$$


For technical reasons that should be clear later, we need to modify the representation as follows: set $1 / \alpha=1 / p-1 / q$ and define:

$$
v(t)=\alpha u\left(t^{\alpha}\right) \quad \text { for } t>0
$$

A simple use of substitution shows that $v(\cdot)$ is a representation of $x$ in the compatible couple $\left(L_{1}(\mathcal{M}), \mathcal{M}\right)$ (also for the compatible couple $\left.\left(L_{p}(\mathcal{M}), L_{q}(\mathcal{M})\right)\right)$. Using the representation $v(\cdot)$, the preceding inequality becomes:

$$
\int_{0}^{\infty} \Phi\left[t^{-1 / p} J\left(t^{1 / p-1 / q}, v\left(t^{1 / p-1 / q}\right) ; L_{p}(\mathcal{M}), L_{q}(\mathcal{M})\right)\right] d t \leq C_{\Phi, p, q} \tau[\Phi(|x|)] .
$$

Next, we discretize the integral in (4.7). If we set $v_{v}=\int_{2^{v}}^{2^{v+1}} v(t) d t / t$ for every $v \in \mathbb{Z}$, then $v_{v} \in L_{1}(\mathcal{M}) \cap \mathcal{M}$ and

$$
x=\sum_{\nu \in \mathbb{Z}} v_{v} \quad\left(\text { convergence in } L_{p}(\mathcal{M})+L_{q}(\mathcal{M})\right) .
$$

By Lemma 3.12(i), we deduce from (4.7) that if $\theta=1 / p-1 / q$ then

$$
\sum_{\nu \in \mathbb{Z}} 2^{\nu / \theta} \Phi\left[2^{-v /(\theta p)} J\left(2^{\nu}, v_{\nu} ; L_{p}(\mathcal{M}), L_{q}(\mathcal{M})\right)\right] \leq C_{\Phi, p, q} \tau[\Phi(|x|)]
$$

Step 3. Use of the simultaneous decompositions. In this step, we use the simultaneous decomposition to generate the appropriate decomposition of $x$. This is a reminiscent of an argument used in [43] (see also [44,45]).

For each $v \in \mathbb{Z}$, we note that since $v_{v} \in L_{1}(\mathcal{M}) \cap \mathcal{M}$, Theorem 2.5 applies to $v_{v}$. That is, there exist $a_{v}, b_{v}$, and $c_{v}$ in $L_{p}(\mathcal{M}) \cap L_{q}(\mathcal{M})$ satisfying:

$$
v_{v}=a_{v}+b_{v}+c_{v}
$$

and if $s$ is equal to either $p$ or $q$, then

$$
\left\|a_{v}\right\|_{\mathrm{h}_{s}^{d}}+\left\|b_{v}\right\|_{\mathrm{h}_{s}^{c}}+\left\|c_{v}\right\|_{\mathrm{h}_{s}^{r}} \leq \kappa(p, q)\left\|v_{v}\right\|_{s}
$$

where $\kappa(p, q)=\max \left\{\kappa_{p}, \kappa_{q}\right\}$ with $\kappa_{p}$ and $\kappa_{q}$ are constants from Theorem 2.5. For convenience, we let

$$
\bar{A}:=\left(L_{p}\left(\mathcal{M} \bar{\otimes} \ell_{\infty}\right), L_{q}\left(\mathcal{M} \bar{\otimes} \ell_{\infty}\right)\right) \quad \text { and } \quad \bar{B}:=\left(L_{p}\left(\mathcal{M} \bar{\otimes} B\left(\ell_{2}\left(\mathbb{N}^{2}\right)\right)\right), L_{q}\left(\mathcal{M} \bar{\otimes} B\left(\ell_{2}\left(\mathbb{N}^{2}\right)\right)\right)\right)
$$

For any given $v \in \mathbb{Z}$, we consider the sequences $\mathcal{D}_{d}\left(a_{v}\right) \in \Delta(\bar{A}), U \mathcal{D}_{c}\left(b_{v}\right) \in \Delta(\bar{B})$, and $U \mathcal{D}_{c}\left(c_{v}^{*}\right) \in \Delta(\bar{B})$. We make the crucial observation that the inequalities in (4.11) can be reinterpreted using the $J$-functionals as follows:

$$
\begin{array}{ll}
J\left(t, \mathcal{D}_{d}\left(a_{v}\right) ; \bar{A}\right) \leq \kappa(p, q) J\left(t, v_{v} ; L_{p}(\mathcal{M}), L_{q}(\mathcal{M})\right), & t>0, \\
J\left(t, U \mathcal{D}_{c}\left(b_{v}\right) ; \bar{B}\right) \leq \kappa(p, q) J\left(t, v_{v} ; L_{p}(\mathcal{M}), L_{q}(\mathcal{M})\right), & t>0, \\
J\left(t, U \mathcal{D}_{c}\left(c_{v}^{*}\right) ; \bar{B}\right) \leq \kappa(p, q) J\left(t, v_{v} ; L_{p}(\mathcal{M}), L_{q}(\mathcal{M})\right), & t>0 .
\end{array}
$$

We need the following properties of the three sequences $\left\{\mathcal{D}_{d}\left(a_{v}\right)\right\}_{v \in \mathbb{Z}},\left\{U \mathcal{D}_{c}\left(b_{v}\right)\right\}_{v \in \mathbb{Z}}$, and $\left\{U \mathcal{D}_{c}\left(c_{v}^{*}\right)\right\}_{v \in \mathbb{Z}}$.

\section{Sublemma 4.2.}

(1) $\sum_{v \in \mathbb{Z}} \mathcal{D}_{d}\left(a_{v}\right)$ is (unconditionally) convergent in $L_{\Phi}\left(\mathcal{M} \bar{\otimes} \ell_{\infty}\right)$.

(2) $\sum_{v \in \mathbb{Z}} U \mathcal{D}_{c}\left(b_{v}\right)$ is (unconditionally) convergent in $L_{\Phi}\left(\mathcal{M} \otimes B\left(\ell_{2}\left(\mathbb{N}^{2}\right)\right)\right.$ ).

(3) $\sum_{v \in \mathbb{Z}} U \mathcal{D}_{c}\left(c_{v}^{*}\right)$ is (unconditionally) convergent in $L_{\Phi}\left(\mathcal{M} \bar{\otimes} B\left(\ell_{2}\left(\mathbb{N}^{2}\right)\right)\right.$ ). 
The proof of Sublemma 4.2 is identical to that of [45, Sublemma 3.3]. Indeed, the argument used in [45] would show that these series are weakly unconditionally Cauchy but since $L_{\Phi}$ is reflexive these convergences are automatically unconditional (see [10]). We leave the details to the reader. From Sublemma 4.2, we may deduce that there exist $a \in \mathrm{h}_{\Phi}^{d}(\mathcal{M}), b \in \mathrm{h}_{\Phi}^{c}(\mathcal{M})$, and $c \in \mathrm{h}_{\Phi}^{r}(\mathcal{M})$ such that:

$$
\begin{aligned}
& \mathcal{D}_{d}(a):=\sum_{\nu \in \mathbb{Z}} \mathcal{D}_{d}\left(a_{\nu}\right) \in L_{\Phi}\left(\mathcal{M} \bar{\otimes} \ell_{\infty}\right) ; \\
& U \mathcal{D}_{c}(b):=\sum_{\nu \in \mathbb{Z}} U \mathcal{D}_{c}\left(b_{\nu}\right) \in L_{\Phi}\left(\mathcal{M} \bar{\otimes} B\left(\ell_{2}\left(\mathbb{N}^{2}\right)\right)\right) ; \\
& U \mathcal{D}_{c}\left(c^{*}\right):=\sum_{\nu \in \mathbb{Z}} U \mathcal{D}_{c}\left(c_{\nu}^{*}\right) \in L_{\Phi}\left(\mathcal{M} \bar{\otimes} B\left(\ell_{2}\left(\mathbb{N}^{2}\right)\right)\right) .
\end{aligned}
$$

The fact that the sum of the first series belongs to $\mathcal{D}_{d}\left(\mathrm{~h}_{\Phi}^{d}(\mathcal{M})\right)$ is clear since the terms of the series belong to the closed subspace $\mathcal{D}_{d}\left(\mathrm{~h}_{\Phi}^{d}(\mathcal{M})\right)$ and thus the existence of $a \in \mathrm{h}_{\Phi}^{d}(\mathcal{M})$. Similar observations can be made for the other two series. Now, combining (4.9) with (4.12) lead to the following inequalities:

$$
\begin{aligned}
& \sum_{\nu \in \mathbb{Z}} 2^{\nu / \theta} \Phi\left[2^{-v /(\theta p)} J\left(2^{v}, \mathcal{D}_{d}\left(a_{v}\right) ; \bar{A}\right)\right] \leq C_{\Phi, p, q} \tau[\Phi(|x|)] ; \\
& \sum_{v \in \mathbb{Z}} 2^{\nu / \theta} \Phi\left[2^{-v /(\theta p)} J\left(2^{v}, U \mathcal{D}_{c}\left(b_{\nu}\right) ; \bar{B}\right)\right] \leq C_{\Phi, p, q} \tau[\Phi(|x|)] ; \\
& \sum_{v \in \mathbb{Z}} 2^{v / \theta} \Phi\left[2^{-v /(\theta p)} J\left(2^{v}, U \mathcal{D}_{c}\left(c_{\nu}^{*}\right) ; \bar{B}\right)\right] \leq C_{\Phi, p, q} \tau[\Phi(|x|)] .
\end{aligned}
$$

Next, we go back to the continuous case. By setting for $t \in\left[2^{v}, 2^{v+1}\right)$,

$$
\mathcal{D}_{d}(a(t))=\frac{\mathcal{D}_{d}\left(a_{\nu}\right)}{\log 2} \in \Delta(\bar{A}), \quad U \mathcal{D}_{c}(b(t))=\frac{U \mathcal{D}_{c}\left(b_{\nu}\right)}{\log 2} \in \Delta(\bar{B}), \quad \text { and } \quad U \mathcal{D}_{c}\left(c(t)^{*}\right)=\frac{U \mathcal{D}_{c}\left(c_{\nu}^{*}\right)}{\log 2} \in \Delta(\bar{B}),
$$

we obtain that $\mathcal{D}_{d}(a(\cdot))$ is a representation of $\mathcal{D}_{d}(a)$ in the couple $\bar{A}, U \mathcal{D}_{c}(b(\cdot))$ is a representation of $U \mathcal{D}_{c}(b)$ in the couple $\bar{B}$, and $U \mathcal{D}_{c}\left(c(\cdot)^{*}\right)$ is a representation of $U \mathcal{D}_{c}\left(c^{*}\right)$ in the couple $\bar{B}$. Moreover, Lemma 3.12(ii) and (4.14) give integral estimates involving the $J$-functionals:

$$
\begin{aligned}
& \int_{0}^{\infty} \Phi\left[t^{-1 / p} J\left(t^{1 / p-1 / q} ; \mathcal{D}_{d}\left(a\left(t^{1 / p-1 / q}\right)\right) ; \bar{A}\right)\right] d t \leq C_{\Phi, p, q} \tau[\Phi(|x|)], \\
& \int_{0}^{\infty} \Phi\left[t^{-1 / p} J\left(t^{1 / p-1 / q}, U \mathcal{D}_{c}\left(b\left(t^{1 / p-1 / q}\right)\right) ; \bar{B}\right)\right] d t \leq C_{\Phi, p, q} \tau[\Phi(|x|)], \\
& \int_{0}^{\infty} \Phi\left[t^{-1 / p} J\left(t^{1 / p-1 / q}, U \mathcal{D}_{c}\left(c\left(t^{1 / p-1 / q}\right)^{*}\right) ; \bar{B}\right)\right] d t \leq C_{\Phi, p, q} \tau[\Phi(|x|)] .
\end{aligned}
$$

Step 4. Switching back to $K$-functionals. In this step, we rewrite (4.15) using $K$-functionals. Indeed, from Proposition 3.10, we may state that:

$$
\begin{aligned}
& \int_{0}^{\infty} \Phi\left[t^{-1 / p} K\left(t^{1 / p-1 / q}, \mathcal{D}_{d}(a) ; \bar{A}\right)\right] d t \leq C_{\Phi, p, q} \tau[\Phi(|x|)], \\
& \int_{0}^{\infty} \Phi\left[t^{-1 / p} K\left(t^{1 / p-1 / q}, U \mathcal{D}_{c}(b) ; \bar{B}\right)\right] d t \leq C_{\Phi, p, q} \tau[\Phi(|x|)], \\
& \int_{0}^{\infty} \Phi\left[t^{-1 / p} K\left(t^{1 / p-1 / q}, U \mathcal{D}_{c}\left(c^{*}\right) ; \bar{B}\right)\right] d t \leq C_{\Phi, p, q} \tau[\Phi(|x|)] .
\end{aligned}
$$


The final part of the argument is to convert the inequalities in (4.16) to the $\left(L_{1}, L_{\infty}\right)$ type interpolation couples. This is necessary since our initial connections with $\Phi$-moments are with the $K$-functionals relative to the couple $\left(L_{1}, L_{\infty}\right)$. We use Proposition 3.5 for this task. We recall that if $\mathcal{N}$ is an arbitrary semifinite von Neumann algebra equipped with a normal semifinite trace and $\theta_{0}=1-p^{-1}$ and $\theta_{1}=1-q^{-1}$ then $L_{p}(\mathcal{N})$ and $L_{q}(\mathcal{N})$ belong to the class $\mathcal{C}_{K}\left(\theta_{0},\left(L_{1}(\mathcal{N}), \mathcal{N}\right)\right)$ and $\mathcal{C}_{K}\left(\theta_{0},\left(L_{1}(\mathcal{N}), \mathcal{N}\right)\right)$, respectively. Thus, if we set $\mathcal{N}_{1}:=\mathcal{M} \bar{\otimes} \ell_{\infty}$ and $\mathcal{N}_{2}:=$ $\mathcal{M} \bar{\otimes} B\left(\ell_{2}\left(\mathbb{N}^{2}\right)\right)$, then we may deduce from (4.16) and Proposition 3.5 that:

$$
\begin{aligned}
& \int_{0}^{\infty} \Phi\left[t^{-1} K\left(t, \mathcal{D}_{d}(a) ; L_{1}\left(\mathcal{N}_{1}\right), \mathcal{N}_{1}\right)\right] d t \leq C_{\Phi, p, q} \tau[\Phi(|x|)], \\
& \int_{0}^{\infty} \Phi\left[t^{-1} K\left(t, U \mathcal{D}_{c}(b) ; L_{1}\left(\mathcal{N}_{2}\right), \mathcal{N}_{2}\right)\right] d t \leq C_{\Phi, p, q} \tau[\Phi(|x|)], \\
& \int_{0}^{\infty} \Phi\left[t^{-1} K\left(t, U \mathcal{D}_{c}\left(c^{*}\right) ; L_{1}\left(\mathcal{N}_{2}\right), \mathcal{N}_{2}\right)\right] d t \leq C_{\Phi, p, q} \tau[\Phi(|x|)] .
\end{aligned}
$$

Step 5. Converting (4.17) into $\Phi$-moment inequalities. For this, we consider first the diagonal part. We observe that if $\tau \otimes \gamma$ denotes the natural trace of $\mathcal{N}_{1}$ and $a=\sum_{n} d a_{n}$, then

$$
\begin{aligned}
\sum_{n \geq 1} \tau\left(\Phi\left(\left|d a_{n}\right|\right)\right) & =\tau \otimes \gamma\left[\Phi\left(\left|\mathcal{D}_{d}(a)\right|\right)\right] \\
& =\int_{0}^{\infty} \Phi\left[\mu_{t}\left(\mathcal{D}_{d}(a)\right)\right] d t \\
& \leq \int_{0}^{\infty} \Phi\left[t^{-1} K\left(t, \mathcal{D}_{d}(a) ; L_{1}\left(\mathcal{N}_{1}\right), \mathcal{N}_{1}\right)\right] d t \\
& \leq C_{\Phi, p, q} \tau[\Phi(|x|)],
\end{aligned}
$$

where the singular-value in the 2 nd line is taken with respect to $\left(\mathcal{N}_{1}, \tau \otimes \gamma\right)$ and the last inequality comes from (4.17). This establishes the diagonal part.

For the column version, we have the estimates:

$$
\begin{aligned}
\tau\left[\Phi\left(s_{c}(b)\right)\right] & \left.=\tau \otimes \operatorname{Tr}\left[\Phi\left(\mid U \mathcal{D}_{c}(b)\right] \mid\right)\right] \\
& =\int_{0}^{\infty} \Phi\left[\mu_{t}\left(U \mathcal{D}_{c}(b)\right)\right] d t \\
& \leq \int_{0}^{\infty} \Phi\left[t^{-1} K\left(t, U \mathcal{D}_{c}(b) ; L_{1}\left(\mathcal{N}_{2}\right), \mathcal{N}_{2}\right)\right] d t \\
& \leq C_{\Phi, p, q} \tau[\Phi(|x|)]
\end{aligned}
$$

where the first equality comes from (2.8), the singular values are taken relative to $\left(\mathcal{N}_{2}, \tau \otimes \operatorname{Tr}\right)$, and the last inequality is from (4.17). Similarly, we may also deduce that

$$
\tau\left[\Phi\left(s_{r}(c)\right)\right]=\tau\left[\Phi\left(s_{c}\left(c^{*}\right)\right)\right] \leq C_{\Phi, p, q} \tau[\Phi(|x|)] .
$$

By combining the last three estimates, we have

$$
\tau\left[\Phi\left(s_{c}(b)\right)\right]+\tau\left[\Phi\left(s_{r}(c)\right)\right]+\sum_{n \geq 1} \tau\left[\Phi\left(\left|d a_{n}\right|\right)\right] \leq C_{\Phi, p, q} \tau[\Phi(|x|)] .
$$

To conclude the proof, we note from (4.8), (4.10), and (4.13), that the identity $x=a+b+c$ is clear from the construction. This completes the proof for the case $x \in L_{1}(\mathcal{M}) \cap \mathcal{M}$.

- Assume now that $x \in L_{\Phi}(\mathcal{M})$. Since $L_{\Phi}(\mathcal{M})$ is a reflexive space, $L_{1}(\mathcal{M}) \cap \mathcal{M}$ is a dense subset of $L_{\Phi}(\mathcal{M})$. Fix a sequence $\left(x^{(m)}\right)_{m \geq 1}$ in $L_{1}(\mathcal{M}) \cap \mathcal{M}$ such that $\lim _{m \rightarrow \infty}\left\|x^{(m)}-x\right\|_{L_{\Phi}(\mathcal{M})}=0$. By Lemma 2.1 , we also have 
$\lim _{m \rightarrow \infty} \tau\left[\Phi\left(\left|x^{(m)}\right|\right)\right]=\tau[\Phi(|x|)]$. From the previous case, for every $m \geq 1$, there exists a decomposition $x^{(m)}=$ $a^{(m)}+b^{(m)}+c^{(m)}$ with $a^{(m)} \in \mathrm{h}_{\Phi}^{d}(\mathcal{M}), b^{(m)} \in \mathrm{h}_{\Phi}^{c}(\mathcal{M})$, and $c^{(m)} \in \mathrm{h}_{\Phi}^{r}(\mathcal{M})$ that satisfy

$$
\tau\left[\Phi\left(s_{c}\left(b^{(m)}\right)\right)\right]+\tau\left[\Phi\left(s_{r}\left(c^{(m)}\right)\right)\right]+\sum_{n \geq 1} \tau\left[\Phi\left(\left|d a_{n}^{(m)}\right|\right)\right] \leq C_{\Phi, p, q} \tau\left[\Phi\left(\left|x^{(m)}\right|\right)\right] .
$$

From reflexivity, we may assume (by taking subsequence if necessary) that $\left\{\left(a^{(m)}, b^{(m)}, c^{(m)}\right)\right\}_{m \geq 1}$ converges to $(a, b, c)$ for the weak topology in $\mathrm{h}_{\Phi}^{d}(\mathcal{M}) \oplus_{\infty} \mathrm{h}_{\Phi}^{c}(\mathcal{M}) \oplus_{\infty} \mathrm{h}_{\Phi}^{r}(\mathcal{M})$. Clearly, $x=a+b+c$. By Lemma 2.1, we obtain the following inequalities: $\sum_{n \geq 1} \tau\left[\Phi\left(\left|d a_{n}\right|\right)\right] \leq \liminf _{m \rightarrow \infty} \sum_{n \geq 1} \tau\left[\Phi\left(\left|d a_{n}^{(m)}\right|\right)\right], \quad \tau\left[\Phi\left(s_{c}(b)\right)\right] \leq$ $\liminf _{m \rightarrow \infty} \tau\left[\Phi\left(s_{c}\left(b^{(m)}\right)\right)\right]$, and $\tau\left[\Phi\left(s_{r}(c)\right)\right] \leq \liminf _{m \rightarrow \infty} \tau\left[\Phi\left(s_{r}\left(c^{(m)}\right)\right)\right]$. These yield the following estimates:

$$
\begin{aligned}
S(a, b, c ; \Phi): & =\tau\left[\Phi\left(s_{c}(b)\right)\right]+\tau\left[\Phi\left(s_{r}(c)\right)\right]+\sum_{n \geq 1} \tau\left[\Phi\left(\left|d a_{n}\right|\right)\right] \\
& \leq \limsup _{m \rightarrow \infty}\left\{\tau\left[\Phi\left(s_{c}\left(b^{(m)}\right)\right)\right]+\tau\left[\Phi\left(s_{r}\left(c^{(m)}\right)\right)\right]+\sum_{n \geq 1} \tau\left[\Phi\left(\left|d a_{n}^{(m)}\right|\right)\right]\right\} \\
& \leq C_{\Phi, p, q} \lim _{m \rightarrow \infty} \tau\left[\Phi\left(\left|x^{(m)}\right|\right)\right] \\
& =C_{\Phi, p, q} \tau[\Phi(|x|)] .
\end{aligned}
$$

The proof is complete.

Remark 4.3. For the case where $\mathcal{M}$ is a finite von Neumann algebra, it is not necessary in our argument above to separate the particular case where $x \in L_{1}(\mathcal{M}) \cap \mathcal{M}$ from the general case. Indeed, when $\mathcal{M}$ is finite, $L_{\Phi}(\mathcal{M}) \subset$ $L_{r}(\mathcal{M})$ whenever $p<r<p_{\Phi}$, thus the argument used in Step 2 applies directly to any element of $L_{\Phi}(\mathcal{M})$.

Our next result deals with the case where the indices of the Orlicz function $\Phi$ are larger than 2. It may be viewed as a common generalization of a $\Phi$-moment result from classical martingale theory [36, Theorem 1] and the noncommutative Burkholder inequalities from [23, Theorem 5.1].

Theorem 4.4. Let $\Phi$ be an Orlicz function satisfying $2<p_{\Phi} \leq q_{\Phi}<\infty$. There exist positive constants $\delta_{\Phi}$ and $\eta_{\Phi}$ depending only on $\Phi$ such that for every martingale $x \in L_{\Phi}(\mathcal{M})$, the following inequalities hold:

$$
\delta_{\Phi}^{-1} M_{\Phi}(x) \leq \tau[\Phi(|x|)] \leq \eta_{\Phi} M_{\Phi}(x),
$$

where $M_{\Phi}(x)=\max \left\{\sum_{n \geq 1} \tau\left[\Phi\left(\left|d x_{n}\right|\right)\right], \tau\left[\Phi\left(s_{c}(x)\right)\right], \tau\left[\Phi\left(s_{r}(x)\right)\right]\right\}$.

Proof. We begin with the first inequality. This is a simple application of Proposition 3.3 and the noncommutative Burkholder inequalities. We leave the details to the reader.

The proof for the second inequality is more involved. Our approach is a duality type argument based on the first inequality in Theorem 4.1 and Proposition 2.3. Let $\Phi^{*}$ denote the Orlicz function complementary to $\Phi$. First, we note that since $2<p_{\Phi} \leq q_{\Phi}<\infty$, it follows that $1<p_{\Phi^{*}} \leq q_{\Phi^{*}}<2$. In particular, Theorem 4.1 applies to bounded martingales in $L_{\Phi^{*}}(\mathcal{M})$.

Next, we observe that $\lim _{t \rightarrow 0^{+}} M\left(t, \Phi^{*}\right)=0$. This fact can be easily seen from the definitions of the indices. We may choose $t_{\Phi}$ small enough so that

$$
M\left(t_{\Phi}, \Phi^{*}\right) \leq\left(2 \delta_{\Phi^{*}}\right)^{-1}
$$

where $\delta_{\Phi^{*}}$ is the constant from Theorem 4.1 applied to $\Phi^{*}$. This is equivalent to

$$
\Phi^{*}\left(t_{\Phi} s\right) \leq\left(2 \delta_{\Phi^{*}}\right)^{-1} \Phi^{*}(s), \quad s>0 .
$$


Thus, by functional calculus, for any operator $0 \leq z \in L_{\Phi^{*}}(\mathcal{M})$, we have

$$
\Phi^{*}\left(t_{\Phi} z\right) \leq\left(2 \delta_{\Phi^{*}}\right)^{-1} \Phi^{*}(z) .
$$

We are now ready to provide the proof. Assume first that $x \in L_{1}(\mathcal{M}) \cap \mathcal{M}$. By Proposition 2.3, we may choose $0 \leq y \in L_{\Phi^{*}}(\mathcal{M})$ such that $y$ commutes with $|x|$ and

$$
\Phi(|x|)+\Phi^{*}(y)=y|x| .
$$

If $x=u|x|$ is the polar decomposition of $x$, we set $y^{\prime}:=y u^{*} \in L_{\Phi^{*}}(\mathcal{M})$. Applying Theorem 4.1 to $y^{\prime}$, there exists a decomposition $y^{\prime}=y^{c}+y^{r}+y^{d}$ with $y^{c} \in \mathrm{h}_{\Phi^{*}}^{c}(\mathcal{M}), y^{r} \in \mathrm{h}_{\Phi^{*}}^{r}(\mathcal{M})$, and $y^{d} \in \mathrm{h}_{\Phi^{*}}^{d}(\mathcal{M})$ satisfying:

$$
\tau\left[\Phi^{*}\left(s_{c}\left(y^{c}\right)\right)\right]+\tau\left[\Phi^{*}\left(s_{r}\left(y^{r}\right)\right)\right]+\sum_{n \geq 1} \tau\left[\Phi^{*}\left(\left|d y_{n}^{d}\right|\right)\right] \leq 2 \delta_{\Phi^{*}} \tau\left[\Phi^{*}\left(\left|y^{\prime}\right|\right)\right] .
$$

Taking traces on (4.19) together with the decomposition of $y^{\prime}$, we have

$$
\begin{aligned}
\tau[\Phi(|x|)]+\tau\left[\Phi^{*}(y)\right] & =\tau(y|x|) \\
& =\tau\left(y^{\prime} x\right) \\
& =\tau\left(x y^{d}\right)+\tau\left(x y^{c}\right)+\tau\left(x y^{r}\right) \\
& :=I+I I+I I I .
\end{aligned}
$$

We estimate $I, I I$, and $I I I$ separately. First, by applying Lemma 2.2 and (4.18), we get the following estimates:

$$
\begin{aligned}
I & =\sum_{n \geq 1} \tau\left(d x_{n} d y_{n}^{d}\right) \\
& \leq \sum_{n \geq 1} \tau\left[\Phi\left(t_{\Phi}^{-1}\left|d x_{n}\right|\right)\right]+\tau\left[\Phi^{*}\left(t_{\Phi}\left|d y_{n}^{d}\right|\right)\right] \\
& \leq \sum_{n \geq 1} \tau\left[\Phi\left(t_{\Phi}^{-1}\left|d x_{n}\right|\right)\right]+\left(2 \delta_{\Phi^{*}}\right)^{-1} \sum_{n \geq 1} \tau\left[\Phi^{*}\left(\left|d y_{n}^{d}\right|\right)\right] .
\end{aligned}
$$

To estimate $I I$, we use the embedding of $h_{\Phi}^{c}(\mathcal{M})$ into $L_{\Phi}\left(\mathcal{M} \bar{\otimes} B\left(\ell_{2}\left(\mathbb{N}^{2}\right)\right)\right)$. First, we note that since the conditional expectations $\mathcal{E}_{k}$ 's are trace preserving, we have

$$
I I=\sum_{n \geq 1} \tau\left(\mathcal{E}_{n-1}\left(d x_{n} d y_{n}^{c}\right)\right)=\tau\left(\sum_{n \geq 1} \mathcal{E}_{n-1}\left(d x_{n} d y_{n}^{c}\right)\right) .
$$

We should note here that since $x \in L_{1}(\mathcal{M}) \cap \mathcal{M}$, for every $n \geq 1, d x_{n} d y_{n}^{c} \in L_{1}(\mathcal{M})+\mathcal{M}$ and therefore $\sum_{n \geq 1} \mathcal{E}_{n-1}\left(d x_{n} d y_{n}^{c}\right)$ is a well-defined operator that belongs to $L_{1}(\mathcal{M})$. We claim that

$$
I I=\tau \otimes \operatorname{Tr}\left[U \mathcal{D}_{c}\left(x^{*}\right)^{*} U \mathcal{D}_{c}\left(y^{c}\right)\right] .
$$

To verify this claim, we begin with the fact taken from Lemma 3.2 that $\mathrm{h}_{\Phi^{*}}^{c}(\mathcal{M}) \subseteq \mathrm{h}_{p}^{c}(\mathcal{M})+\mathrm{h}_{q}^{c}(\mathcal{M})$ where $1<p<$ $p_{\Phi^{*}} \leq q_{\Phi^{*}}<q<2$. Write $y^{c}=\alpha^{c}+\beta^{c}$ where $\alpha^{c} \in \mathrm{h}_{p}^{c}(\mathcal{M})$ and $\beta^{c} \in \mathrm{h}_{q}^{c}(\mathcal{M})$. Then from (2.5), we have

$$
\left(\sum_{n \geq 1} \mathcal{E}_{n-1}\left(d x_{n} d \alpha_{n}^{c}\right)\right) \otimes e_{1,1} \otimes e_{1,1}=U \mathcal{D}_{c}\left(x^{*}\right)^{*} U \mathcal{D}_{c}\left(\alpha^{c}\right)
$$

and

$$
\left(\sum_{n \geq 1} \mathcal{E}_{n-1}\left(d x_{n} d \beta_{n}^{c}\right)\right) \otimes e_{1,1} \otimes e_{1,1}=U \mathcal{D}_{c}\left(x^{*}\right)^{*} U \mathcal{D}_{c}\left(\beta^{c}\right) .
$$


Taking the sum of the above two equalities clearly shows the claim.

As in the case of $I$, by applying Lemma 2.2 together with (4.18), we obtain the estimates

$$
\begin{aligned}
I I & =\tau \otimes \operatorname{Tr}\left[U \mathcal{D}_{c}\left(x^{*}\right)^{*} U \mathcal{D}_{c}\left(y^{c}\right)\right] \\
& \leq \tau \otimes \operatorname{Tr}\left[\Phi\left(t_{\Phi}^{-1}\left|U \mathcal{D}_{c}\left(x^{*}\right)\right|\right)\right]+\tau \otimes \operatorname{Tr}\left[\Phi^{*}\left(t_{\Phi}\left|U \mathcal{D}_{c}\left(y^{c}\right)\right|\right)\right] \\
& =\tau\left[\Phi\left(t_{\Phi}^{-1} s_{r}(x)\right)\right]+\tau\left[\Phi^{*}\left(t_{\Phi} s_{c}\left(y^{c}\right)\right)\right] \\
& \leq \tau\left[\Phi\left(t_{\Phi}^{-1} s_{r}(x)\right)\right]+\left(2 \delta_{\Phi^{*}}\right)^{-1} \tau\left[\Phi^{*}\left(s_{c}\left(y^{c}\right)\right)\right] .
\end{aligned}
$$

By repeating the same argument with $y^{r}$, we may also state that

$$
I I I \leq \tau\left[\Phi\left(t_{\Phi}^{-1} s_{c}(x)\right)\right]+\left(2 \delta_{\Phi^{*}}\right)^{-1} \tau\left[\Phi^{*}\left(s_{r}\left(y^{r}\right)\right)\right] .
$$

Taking the summation of the previous estimates and applying (4.20), we obtain that

$$
\begin{aligned}
\tau[\Phi(|x|)]+\tau\left[\Phi^{*}(y)\right] \leq & \sum_{n \geq 1} \tau\left[\Phi\left(t_{\Phi}^{-1}\left|d x_{n}\right|\right)\right]+\tau\left[\Phi\left(t_{\Phi}^{-1} s_{c}(x)\right)\right] \\
& +\tau\left[\Phi\left(t_{\Phi}^{-1} s_{r}(x)\right)\right]+\tau\left[\Phi^{*}\left(\left|y^{\prime}\right|\right)\right] .
\end{aligned}
$$

But since $\tau\left[\Phi^{*}\left(\left|y^{\prime}\right|\right)\right]=\int_{0}^{\infty} \Phi^{*}\left(\mu_{t}\left(y u^{*}\right)\right) d t \leq \int_{0}^{\infty} \Phi^{*}\left(\mu_{t}(y)\right) d t=\tau\left[\Phi^{*}(y)\right]$, we deduce that

$$
\begin{aligned}
\tau[\Phi(|x|)] & \leq \sum_{n \geq 1} \tau\left[\Phi\left(t_{\Phi}^{-1}\left|d x_{n}\right|\right)\right]+\tau\left[\Phi\left(t_{\Phi}^{-1} s_{c}(x)\right)\right]+\tau\left[\Phi\left(t_{\Phi}^{-1} s_{r}(x)\right)\right] \\
& \leq 3 \max \left\{\sum_{n \geq 1} \tau\left[\Phi\left(t_{\Phi}^{-1}\left|d x_{n}\right|\right)\right], \tau\left[\Phi\left(t_{\Phi}^{-1} s_{c}(x)\right)\right], \tau\left[\Phi\left(t_{\Phi}^{-1} s_{r}(x)\right)\right]\right\} .
\end{aligned}
$$

The existence of the constant $\eta_{\Phi}$ and the second inequality in $\left(B_{\Phi}\right)$ now follow from the $\Delta_{2}$-condition. Thus, we have shown the second inequality in $\left(B_{\Phi}\right)$ for $x \in L_{1}(\mathcal{M}) \cap \mathcal{M}$. The proof for the general case follows the same line of reasoning as in the last part of the proof of Theorem 4.1 so we omit the details.

We should point out that the approach used in [23] for the $p$ th moments was to establish the case $2<p<\infty$ first and then deduce the case $1<p<2$ by duality. At the time of this writing, we do not know of any direct proof of Theorem 4.4. This is rather surprising since the case $p>2$ is in general more accessible than the case $1<p<2$. It seems that the existing argument for $p$ th moment $(p>2)$ from [23] can be adapted to $\Phi$-moment only for the case where the Orlicz function $\Phi$ satisfies a Hölder type inequality $\Phi(t s) \leq C \Phi\left(t^{2}\right)^{1 / 2} \Phi\left(s^{2}\right)^{1 / 2}$ for every $t, s>0$ and $C$ is an absolute constant. The above condition is clearly satisfied by power functions and functions of the type $\Phi(t)=t^{\alpha} e^{\beta t}$ with $\alpha>1$ and $\beta>0$ among others. It is however a stronger condition than being just submultiplicative. We also do not know whether Theorem 4.1 can be derived from Theorem 4.4 via Proposition 2.3.

\section{5. $\Phi$-Moments and noncommutative Rosenthal inequalities}

In this section, we consider notions of noncommutative independences introduced in [25] and discuss corresponding $\Phi$-moment results for sums of independent sequences.

Throughout, we assume that $\mathcal{N}$ and $\mathcal{A}_{n}$ 's are von Neumann subalgebras of $(\mathcal{M}, \tau)$ with $\mathcal{N} \subset \mathcal{A}_{n}$ for all $n \geq 1$. We further assume that there exist trace preserving normal conditional expectations $\mathcal{E}_{\mathcal{N}}: \mathcal{M} \rightarrow \mathcal{N}$ and $\mathcal{E}_{\mathcal{A}_{n}}: \mathcal{M} \rightarrow \mathcal{A}_{n}$ for all $n \geq 1$. Following [25], we consider the following notions of independences:

\section{Definition 5.1.}

(i) We say that $\left(\mathcal{A}_{n}\right)_{n \geq 1}$ are independent over $\mathcal{N}$ (or with respect to $\mathcal{E}_{\mathcal{N}}$ ) if for every $n \geq 1, \mathcal{E}_{\mathcal{N}}(x y)=\mathcal{E}_{\mathcal{N}}(x) \mathcal{E}_{\mathcal{N}}(y)$ holds for all $x \in \mathcal{A}_{n}$ and $y$ in the von Neumann algebra generated by $\left(\mathcal{A}_{j}\right)_{j \neq n}$. 
(ii) We say that the sequence $\left(\mathcal{A}_{n}\right)_{n \geq 1}$ is order independent over $\mathcal{N}$ (or with respect to $\mathcal{E}_{\mathcal{N}}$ ) if for every $n \geq 2$,

$$
\mathcal{E}_{V N\left(\mathcal{A}_{1}, \ldots, \mathcal{A}_{n-1}\right)}(x)=\mathcal{E}_{\mathcal{N}}(x), \quad x \in \mathcal{A}_{n},
$$

where $\mathcal{E}_{V N\left(\mathcal{A}_{1}, \ldots, \mathcal{A}_{n-1}\right)}$ denotes the normal conditional expectation onto the von Neumann subalgebra degenerated by $\mathcal{A}_{1}, \ldots, \mathcal{A}_{n-1}$.

(iii) A sequence $\left(a_{n}\right)_{n \geq 1}$ in $L_{1}(\mathcal{M})+\mathcal{M}$ is called (order) independent with respect to $\mathcal{E}_{\mathcal{N}}$ if there is an (order) independent sequence $\left(\mathcal{A}_{n}\right)_{n \geq 1}$ of von Neumann subalgebras of $\mathcal{M}$ such that $a_{n} \in L_{1}\left(\mathcal{A}_{n}\right)+\mathcal{A}_{n}$ for all $n \geq 1$.

It was noted in [25] (Lemma 1.2) that independence implies order independence. We refer to [25] for extensive stud-

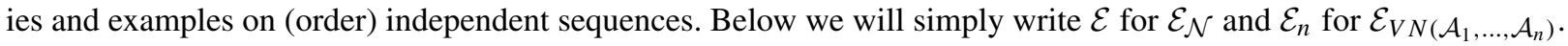

It is important to observe that if $\left(\mathcal{A}_{n}\right)_{n \geq 1}$ is an order independent sequence of von Neumann subalgebras and $a_{n} \in L_{p}\left(\mathcal{A}_{n}\right)$ with $\mathcal{E}\left(a_{n}\right)=0(n \geq 1)$ then $\left(a_{n}\right)_{n \geq 1}$ is a martingale difference sequence with respect to the increasing filtration $\left(V N\left(\mathcal{A}_{1}, \ldots, \mathcal{A}_{n}\right)\right)_{n \geq 1}$. In this case, one clearly see from the definition that when $p \geq 2$, the row and column conditioned square functions take the following simpler forms:

$$
s_{c}\left(\sum_{n \geq 1} a_{n}\right)=\left(\sum_{n \geq 1} \mathcal{E}\left(a_{n}^{*} a_{n}\right)\right)^{1 / 2} \text { and } s_{r}\left(\sum_{n \geq 1} a_{n}\right)=\left(\sum_{n \geq 1} \mathcal{E}\left(a_{n} a_{n}^{*}\right)\right)^{1 / 2} .
$$

For the remaining of this section, any reference to martingales is understood to be with respect to the filtration described above.

From the preceding discussion, for the special case of sums of order independent sequences, Theorem 4.4 reads as follows:

Corollary 5.2. Let $\Phi$ be an Orlicz function satisfying $2<p_{\Phi} \leq q_{\Phi}<\infty$. There exist positive constants $\delta_{\Phi}$ and $\eta_{\Phi}$ depending only on $\Phi$ such that for every order independent sequence $\left(a_{n}\right)_{n \geq 1} \subset L_{\Phi}(\mathcal{M})$ with $\mathcal{E}\left(a_{n}\right)=0$, the following inequalities hold:

$$
\delta_{\Phi}^{-1} M_{\Phi}(a) \leq \tau\left[\Phi\left(\left|\sum_{n \geq 1} a_{n}\right|\right)\right] \leq \eta_{\Phi} M_{\Phi}(a),
$$

where $M_{\Phi}(a)=\max \left\{\sum_{n \geq 1} \tau\left[\Phi\left(\left|a_{n}\right|\right)\right], \tau\left[\Phi\left(\left(\sum_{n \geq 1} \mathcal{E}\left(a_{n}^{*} a_{n}\right)\right)^{1 / 2}\right)\right], \tau\left[\Phi\left(\left(\sum_{n \geq 1} \mathcal{E}\left(a_{n} a_{n}^{*}\right)\right)^{1 / 2}\right)\right]\right\}$.

However, when $1<p_{\Phi} \leq q_{\Phi}<2$, the case of sums of independent sequences can not be directly read from Theorem 4.1 since the decomposition we have in the statement of Theorem 4.1 is not necessarily made up of independent sequences. Handling this case requires a way of modifying martingale difference sequences into independent sequences. Below, we adapt the approach of [25] for this reduction. In order to state our results, we need to formally introduce some new notation.

For any finite sequence $\left(a_{n}\right)_{1 \leq n \leq N} \in \mathcal{F}$, we define

$$
\left\|\left(a_{n}\right)_{1 \leq n \leq N}\right\|_{L_{\Phi}\left(\mathcal{M}, \mathcal{E}, \ell_{2}^{c}\right)}:=\left\|\left(\sum_{n=1}^{N} \mathcal{E}\left(a_{k}^{*} a_{k}\right)\right)^{1 / 2}\right\|_{L_{\Phi}(\mathcal{M})} .
$$

If we set $\bar{a}=\sum_{n=1}^{N} e_{n, 1} \otimes a_{n} \in L_{1}\left(B\left(\ell_{2}^{N}\right) \otimes \mathcal{M}\right) \cap\left(B\left(\ell_{2}^{N}\right) \otimes \mathcal{M}\right)$ and $\widetilde{\mathcal{E}}=I d \otimes \mathcal{E}$, then we have

$$
\left\|\left(a_{n}\right)_{1 \leq n \leq N}\right\|_{L_{\Phi}\left(\mathcal{M}, \mathcal{E}, \ell_{2}^{c}\right)}=\|\bar{a}\|_{L_{\Phi}\left(B\left(\ell_{2}^{N}\right) \otimes \mathcal{M}, \widetilde{\mathcal{E}}\right)},
$$

where $L_{\Phi}\left(B\left(\ell_{2}^{N}\right) \otimes \mathcal{M}, \widetilde{\mathcal{E}}\right)$ is the conditioned space introduced in Section 2 . Therefore, $\|\cdot\|_{L_{\Phi}\left(\mathcal{M}, \mathcal{E}, \ell_{2}^{c}\right)}$ defines a norm on the linear space $\mathcal{F}$. We define $L_{\Phi}\left(\mathcal{M}, \mathcal{E}, \ell_{2}^{c}\right)$ to be the completion of the space $\left(\mathcal{F},\|\cdot\|_{L_{\Phi}\left(\mathcal{M}, \mathcal{E}, \ell_{2}^{c}\right)}\right)$. The space $L_{\Phi}\left(\mathcal{M}, \mathcal{E}, \ell_{2}^{r}\right)$ is defined in a similar way.

Now, we consider the subspace $\mathcal{F}^{\text {(Ind) }}$ of $\mathcal{F}$ consisting of all sequences $\left(a_{n}\right)_{n \geq 1}$ in $\mathcal{F}$ such that $a_{n} \in L_{1}\left(\mathcal{A}_{n}\right) \cap \mathcal{A}_{n}$ and $\mathcal{E}\left(a_{n}\right)=0$ and let $\mathcal{R}_{\Phi}^{c}(\mathcal{M})$ be the closure of $\mathcal{F}^{(\text {Ind) }}$ in $L_{\Phi}\left(\mathcal{M}, \mathcal{E}, \ell_{2}^{c}\right)$. Similarly, we may define the corresponding 
subspaces of $L_{\Phi}\left(\mathcal{M}, \mathcal{E}, \ell_{2}^{r}\right)$ and $L_{\Phi}\left(\mathcal{M} \bar{\otimes} \ell_{\infty}\right)$ which will be denoted by $\mathcal{R}_{\Phi}^{r}(\mathcal{M})$ and $\mathcal{R}_{\Phi}^{d}(\mathcal{M})$, respectively. When $\Phi(t)=t^{p}$, these are exactly the spaces $\mathcal{R}_{p}^{c}(\mathcal{M}), \mathcal{R}_{p}^{r}(\mathcal{M})$, and $\mathcal{R}_{p}^{d}(\mathcal{M})$ introduced in [23,25]. If we denote by $\mathrm{J}$ : $\mathcal{F}^{(\text {Ind) }} \rightarrow \mathcal{F}_{M}$ the map defined by $\left(a_{n}\right)_{n \geq 1} \mapsto \sum_{n \geq 1} a_{n}$, then for $s \in\{d, c, r\}$, it extends to an isometric embedding $\mathrm{J}_{\Phi}^{s}: \mathcal{R}_{\Phi}^{s}(\mathcal{M}) \rightarrow \mathrm{h}_{\Phi}^{s}(\mathcal{M})$. Next, we consider the linear map $\Theta: \mathcal{F}_{M} \rightarrow \mathcal{F}^{(\text {Ind) }}$ defined by setting for any given $x \in \mathcal{F}_{M}$,

$$
\Theta(x)_{n}:= \begin{cases}0 & \text { if } n=1 \\ \mathcal{E}_{\mathcal{A}_{n}}\left(d x_{n}\right) & \text { if } n \geq 2\end{cases}
$$

It is clear that for every $n \geq 1, \mathcal{E}\left(\Theta(x)_{n}\right)=0$ and therefore $\Theta(x) \in \mathcal{F}^{\text {(Ind) }}$. The following result is our main tool in the proof of Theorem 5.6 below.

Proposition 5.3. Let $\Phi$ be an Orlicz function with $1<p_{\Phi} \leq q_{\Phi}<\infty$. Then for $s \in\{d, c, r\}, \Theta: h_{\Phi}^{s}(\mathcal{M}) \rightarrow \mathcal{R} \mathcal{R}_{\Phi}^{s}(\mathcal{M})$ is bounded. Moreover, there exists a constant $C_{\Phi}$ such that for every $x \in \mathrm{h}_{\Phi}^{d}(\mathcal{M})\left(\right.$ respectively, $y \in \mathrm{h}_{\Phi}^{c}(\mathcal{M})$ ),

$$
\sum_{n \geq 2} \tau\left[\Phi\left(\left|\Theta(x)_{n}\right|\right)\right] \leq C_{\Phi} \sum_{n \geq 1} \tau\left[\Phi\left(\left|d x_{n}\right|\right)\right],
$$

respectively,

$$
\tau\left[\Phi\left(s_{c}\left(\sum_{n \geq 2} \Theta(y)_{n}\right)\right)\right] \leq C_{\Phi} \tau\left[\Phi\left(s_{c}(y)\right)\right] .
$$

We begin with the verification of the following particular case:

Lemma 5.4. Let $1<p<\infty$. Then for $s \in\{d, c, r\}, \Theta: \mathrm{h}_{p}^{s}(\mathcal{M}) \rightarrow \mathcal{R}_{p}^{s}(\mathcal{M})$ is a contraction.

Proof. The diagonal part is trivial from the boundedness of conditional expectations in $L_{p}(\mathcal{M})$ so it suffices to verify the statement for the column version. We use the fact that $L_{p}\left(\mathcal{M}, \mathcal{E}, \ell_{2}^{c}\right)^{*}=L_{p^{\prime}}\left(\mathcal{M}, \mathcal{E}, \ell_{2}^{c}\right)$ where $p^{\prime}$ denotes the index conjugate to $p$ (see [25, Lemma 0.1]). Let $x \in \mathrm{h}_{p}^{c}(\mathcal{M})$ and fix a sequence $\left(v_{n}\right)$ from the unit ball of $L_{p^{\prime}}\left(\mathcal{M}, \mathcal{E}, \ell_{2}^{c}\right)$ (with $v_{1}=0$ ) so that

$$
\|\Theta(x)\|_{\mathcal{R}_{p}^{c}}=\|\Theta(x)\|_{L_{p}\left(\mathcal{M}, \mathcal{E}, \ell_{2}^{c}\right)}=\sum_{n \geq 2} \tau\left(\Theta(x)_{n} v_{n}^{*}\right)=\sum_{n \geq 2} \tau\left(\mathcal{E}_{\mathcal{A}_{n}}\left(d x_{n}\right) v_{n}^{*}\right) .
$$

By trace invariance and duality between $\mathrm{h}_{p}^{c}(\mathcal{M})$ and $\mathrm{h}_{p^{\prime}}^{c}(\mathcal{M})$, we have

$$
\begin{aligned}
\|\Theta(x)\|_{\mathcal{R}_{p}^{c}} & =\sum_{n \geq 2} \tau\left(d x_{n}\left[\mathcal{E}_{\mathcal{A}_{n}}\left(v_{n}^{*}\right)-\mathcal{E}\left(v_{n}^{*}\right)\right]\right) \\
& \leq\left\|\sum_{n \geq 2} d x_{n}\right\|_{\mathrm{h}_{p}^{c}} \cdot\left\|\sum_{n \geq 2} \mathcal{E}_{\mathcal{A}_{n}}\left(v_{n}\right)-\mathcal{E}\left(v_{n}\right)\right\|_{h_{p^{\prime}}^{c}} .
\end{aligned}
$$

One can easily see by independence that for any $n \geq 2$, the following holds:

$$
\mathcal{E}_{n-1}\left|\mathcal{E}_{\mathcal{A}_{n}}\left(v_{n}\right)-\mathcal{E}\left(v_{n}\right)\right|^{2}=\mathcal{E}\left[\mathcal{E}_{\mathcal{A}_{n}}\left(v_{n}\right)^{*} \mathcal{E}_{\mathcal{A}_{n}}\left(v_{n}\right)\right]-\mathcal{E}\left(v_{n}\right)^{*} \mathcal{E}\left(v_{n}\right) \leq \mathcal{E}\left(v_{n}^{*} v_{n}\right) .
$$

This implies in particular that

$$
\left\|\sum_{n \geq 2} \mathcal{E}_{\mathcal{A}_{n}}\left(v_{n}\right)-\mathcal{E}\left(v_{n}\right)\right\|_{h_{p^{\prime}}^{c}} \leq\left\|\left(v_{n}\right)\right\|_{L_{p^{\prime}}\left(\mathcal{M}, \mathcal{E}, \ell_{2}^{c}\right)} \leq 1 .
$$

We deduce that $\|\Theta(x)\|_{\mathcal{R}_{p}^{c}} \leq\left\|\sum_{n \geq 2} d x_{n}\right\|_{\mathrm{h}_{p}^{c}} \leq\|x\|_{\mathrm{h}_{p}^{c}}$ proving that $\Theta$ is a contraction. 
Remark 5.5. In the proof of Lemma 5.4, it is crucial that $v_{1}=0$. Otherwise, we only get the equality $\mathcal{E}_{n-1} \mid \mathcal{E}_{\mathcal{A}_{n}}\left(v_{n}\right)-$ $\left.\mathcal{E}\left(v_{n}\right)\right|^{2}=\left|v_{1}-\mathcal{E}\left(v_{1}\right)\right|^{2}$ when $n=1$. As a result, the estimate $\left\|\sum_{n \geq 1} \mathcal{E}_{\mathcal{A}_{n}}\left(v_{n}\right)-\mathcal{E}\left(v_{n}\right)\right\|_{h_{p^{\prime}}^{c}} \leq\left\|\left(v_{n}\right)\right\|_{L_{p^{\prime}}\left(\mathcal{M}, \mathcal{E}, \ell_{2}^{c}\right)}$ would not be achieved. This is the primary reason for choosing $\Theta(x)_{1}=0$ in the definition of $\Theta$.

The proof of Proposition 5.3 is now a simple interpolation of Lemma 5.4 together with Proposition 3.3. We leave the details to the reader.

The next theorem is our main result for this section. It is a $\Phi$-moment generalization of the noncommutative Rosenthal inequalities from [25, Theorem 3.2].

Theorem 5.6. Let $\Phi$ be an Orlicz function satisfying $1<p_{\Phi} \leq q_{\Phi}<2$. There exist positive constants $\tilde{\delta}_{\Phi}$ and $\tilde{\eta}_{\Phi}$ depending only on $\Phi$ such that for every order independent sequence $\left(x_{n}\right)_{n \geq 1} \subset L_{\Phi}(\mathcal{M})$ with $\mathcal{E}\left(x_{n}\right)=0$, the following inequalities hold:

$$
\tilde{\delta}_{\Phi}^{-1} \widetilde{S}_{\Phi}(x) \leq \tau\left[\Phi\left(\left|\sum_{n \geq 1} x_{n}\right|\right)\right] \leq \tilde{\eta}_{\Phi} \widetilde{S}_{\Phi}(x),
$$

where $\widetilde{S}_{\Phi}(x)=\inf \left\{\tau\left[\Phi\left(s_{c}\left(\sum_{n \geq 1} x_{n}^{c}\right)\right)\right]+\tau\left[\Phi\left(\left(s_{r}\left(\sum_{n \geq 1} x_{n}^{r}\right)\right)\right]+\sum_{n \geq 1} \tau\left[\Phi\left(\left|x_{n}^{d}\right|\right)\right]\right\}\right.$ with the infimum being taken over all $\left(x_{n}^{c}\right) \in \mathcal{R}_{\Phi}^{c}(\mathcal{M}),\left(x_{n}^{r}\right) \in \mathcal{R}_{\Phi}^{r}(\mathcal{M})$, and $\left(x_{n}^{d}\right) \in \mathcal{R}_{\Phi}^{\bar{d}}(\mathcal{M})$ such that for every $n \geq 1, x_{n}=x_{n}^{c}+x_{n}^{r}+x_{n}^{d}$.

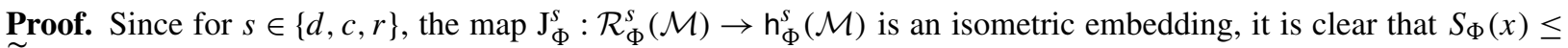
$\widetilde{S}_{\Phi}(x)$. Thus, the second inequality follows immediately from Theorem 4.1.

The proof of the first inequality is a combination of Theorem 4.1 and Proposition 5.3. First, by Theorem 4.1, there exists a constant $\delta_{\Phi}$ such that if $\left(x_{n}\right)$ is as in the statement of the theorem then there exists a decomposition $x_{n}=d \alpha_{n}+d \beta_{n}+d \gamma_{n}$ where $\alpha \in \mathrm{h}_{\Phi}^{d}(\mathcal{M}), \beta \in \mathrm{h}_{\Phi}^{c}(\mathcal{M}), \gamma \in \mathrm{h}_{\Phi}^{r}(\mathcal{M})$, and

$$
\tau\left[\Phi\left(s_{c}(\beta)\right)\right]+\tau\left[\Phi\left(s_{r}(\gamma)\right)\right]+\sum_{n \geq 1} \tau\left[\Phi\left(\left|d \alpha_{n}\right|\right)\right] \leq 2 \delta_{\Phi} \tau\left[\Phi\left(\left|\sum_{n \geq 1} x_{n}\right|\right)\right] .
$$

Let $x_{1}^{d}=x_{1}$ and $x_{n}^{d}=\Theta(\alpha)_{n}$ for $n \geq 2$. Similarly, let $x^{c}=\Theta(\beta)$ and $x^{r}=\Theta(\gamma)$. Then for every $n \geq 1, x_{n}=$ $x_{n}^{d}+x_{n}^{r}+x_{n}^{r}$. From Proposition 5.3, $x^{d} \in \mathcal{R}_{\Phi}^{d}(\mathcal{M}), x^{c} \in \mathcal{R}_{\Phi}^{c}(\mathcal{M})$, and $x^{r} \in \mathcal{R}_{\Phi}^{r}(\mathcal{M})$. Moreover, there exists a constant $C_{\Phi}$ such that:

$$
\begin{aligned}
& \tau\left[\Phi\left(s_{c}\left(\sum_{n \geq 2} x_{n}^{c}\right)\right)\right]+\tau\left[\Phi\left(s_{r}\left(\sum_{n \geq 2} x_{n}^{r}\right)\right)\right]+\sum_{n \geq 1} \tau\left[\Phi\left(\left|x_{n}^{d}\right|\right)\right] \\
& \leq \tau\left[\Phi\left(\left|x_{1}\right|\right)\right]+C_{\Phi}\left\{\tau\left[\Phi\left(s_{c}(\beta)\right)\right]+\tau\left[\Phi\left(s_{r}(\gamma)\right)\right]+\sum_{n \geq 1} \tau\left[\Phi\left(\left|d \alpha_{n}\right|\right)\right]\right\} \\
& \quad \leq \tau\left[\Phi\left(\left|x_{1}\right|\right)\right]+2 C_{\Phi} \delta_{\Phi} \tau\left[\Phi\left(\left|\sum_{n \geq 1} x_{n}\right|\right)\right] .
\end{aligned}
$$

Since $\mathcal{E}_{\mathcal{A}_{1}}$ is bounded in $L_{\Phi}(\mathcal{M})$, we have $\tau\left[\Phi\left(\left|x_{1}\right|\right)\right] \leq D_{\Phi} \tau\left[\Phi\left(\left|\sum_{n \geq 1} x_{n}\right|\right)\right]$ for some constant $D_{\Phi}$. We conclude that

$$
\tau\left[\Phi\left(s_{c}\left(\sum_{n \geq 2} x_{n}^{c}\right)\right)\right]+\tau\left[\Phi\left(s_{r}\left(\sum_{n \geq 2} x_{n}^{r}\right)\right)\right]+\sum_{n \geq 1} \tau\left[\Phi\left(\left|x_{n}^{d}\right|\right)\right] \leq\left(D_{\Phi}+2 C_{\Phi} \delta_{\Phi}\right) \tau\left[\Phi\left(\left|\sum_{n \geq 1} x_{n}\right|\right)\right] .
$$

This completes the proof.

We take the opportunity to include noncommutative Rosenthal inequalities for the case of noncommutative symmetric spaces. Following [45], we let $E$ denote a symmetric space on $(0, \infty)$ that satisfies the Fatou property. We 
denote by $p_{E}$ and $q_{E}$ the lower and upper Boyd indices respectively. We may repeat verbatim the construction above and define the spaces $\mathcal{R}_{E}^{c}(\mathcal{M}), \mathcal{R}_{E}^{r}(\mathcal{M})$, and $\mathcal{R}_{E}^{d}(\mathcal{M})$ by simply replacing $L_{\Phi}$ with $E$. Obvious modification of the proof of Proposition 5.3 also gives that if $E$ has nontrivial Boyd indices then $\Theta$ extends to a bounded linear map from $\mathrm{h}_{E}^{s}(\mathcal{M})$ into $\mathcal{R}_{E}^{s}(\mathcal{M})$ for $s \in\{d, c, r\}$ whenever $1<p_{E} \leq q_{E}<\infty$. Combining this result with the extension of the Burkholder inequalities to noncommutative symmetric spaces from [45, Theorem 3.1], we may also state the following generalization of [25, Theorem 3.2]:

Theorem 5.7. Let $E$ be a symmetric function space defined on $(0, \infty)$ with the Fatou property and assume that $1<p_{E} \leq q_{E}<2$. Let $x_{n} \in E\left(\mathcal{A}_{n}\right)$ such that $\mathcal{E}\left(x_{n}\right)=0$. Then

$$
\left\|\sum_{n \geq 1} x_{n}\right\|_{E(\mathcal{M})} \simeq_{E} \inf \left\{\left\|\left(x_{n}^{d}\right)\right\|_{\mathcal{R}_{E}^{d}}+\left\|\left(x_{n}^{c}\right)\right\|_{\mathcal{R}_{E}^{c}}+\left\|\left(x_{n}^{r}\right)\right\|_{\mathcal{R}_{E}^{r}}\right\},
$$

where the infimum is taken over all decompositions $x_{n}=x_{n}^{d}+x_{n}^{c}+x_{n}^{r}$ with $\left(x_{n}^{d}\right) \in \mathcal{R}_{E}^{d}(\mathcal{M}),\left(x_{n}^{c}\right) \in \mathcal{R}_{E}^{c}(\mathcal{M})$, and $\left(x_{n}^{r}\right) \in \mathcal{R}_{E}^{r}(\mathcal{M})$.

A version of Theorem 5.7 for the case where the Boyd indices satisfy the condition $2<p_{E} \leq q_{E}<\infty$ was first obtained by Dirksen et al. (see [12, Theorem 6.3]). Similar line of result for martingale BMO-norms of sums of noncommuting independent sequences were also considered in [44, Theorem 5.3]. Other Rosenthal-type inequalities for sums of freely independent sequences can also be found in [18,21,47].

As illustrations, we observe that all examples treated in [25, Section 3] can be easily adapted to Corollary 5.2, Theorem 5.6, and Theorem 5.7. As a sample result, we state the $\Phi$-moment generalization of [25, Theorem 3.3]:

Theorem 5.8. Let $\Phi$ be an Orlicz function and $\left(x_{i j}\right)$ be a finite matrix with entries in $L_{\Phi}(\mathcal{M})$. Assume that the $x_{i j}$ 's are independent with respect to $\mathcal{E}$ and $\mathcal{E}\left(x_{i j}\right)=0$. Then

- for $1<p_{\Phi} \leq q_{\Phi}<2$,

$$
\begin{aligned}
\tau & \otimes \operatorname{tr}\left[\Phi\left(\left|\sum_{i j} x_{i j} \otimes e_{i j}\right|\right)\right] \\
& \simeq_{\Phi} \inf \left\{\sum_{i j} \tau\left[\Phi\left(\left|x_{i j}^{d}\right|\right)\right]+\sum_{j} \tau\left[\Phi\left(\left[\sum_{i} \mathcal{E}\left(\left|x_{i j}^{c}\right|^{2}\right)\right]^{1 / 2}\right)\right]+\sum_{i} \tau\left[\Phi\left(\left[\sum_{j} \mathcal{E}\left(\left|x_{i j}^{r *}\right|^{2}\right)\right]^{1 / 2}\right)\right]\right\},
\end{aligned}
$$

where the infimum is taken over all decompositions $x_{i j}=x_{i j}^{d}+x_{i j}^{c}+x_{i j}^{r}$ with mean zero elements $x_{i j}^{d}, x_{i j}^{c}$, and $x_{i j}^{r}$, which, for each couple $(i, j)$, belong to the Orlicz space associated with the von Neumann algebra generated by $x_{i j}$.

- for $2<p_{\Phi} \leq q_{\Phi}<\infty$,

$$
\begin{aligned}
\tau & \otimes \operatorname{tr}\left[\Phi\left(\left|\sum_{i j} x_{i j} \otimes e_{i j}\right|\right)\right] \\
& \simeq_{\Phi} \max \left\{\sum_{i j} \tau\left[\Phi\left(\left|x_{i j}\right|\right)\right], \sum_{j} \tau\left[\Phi\left(\left[\sum_{i} \mathcal{E}\left(\left|x_{i j}\right|^{2}\right)\right]^{1 / 2}\right)\right], \sum_{i} \tau\left[\Phi\left(\left[\sum_{j} \mathcal{E}\left(\left|x_{i j}^{*}\right|^{2}\right)\right]^{1 / 2}\right)\right]\right\} .
\end{aligned}
$$

As in the case of $p$ th moments, if the von algebra $\mathcal{M}$ is taken to be the $L_{\infty}$-space defined on a probability space, then Theorem 5.8 becomes a statement about $\Phi$-moment inequalities of random matrices.

We conclude this section by noting that by applying the reduction technique used above to the simultaneous decomposition stated in Theorem 2.5, we may also achieve the following version for independent sequences:

Proposition 5.9. There exists a family of constants $\left\{\kappa_{p}^{\prime}: 1<p<2\right\} \subset \mathbb{R}_{+}$satisfying the following: if $\left(x_{n}\right)_{n \geq 1}$ is an order independent sequence in $L_{1}(\mathcal{M}) \cap L_{2}(\mathcal{M})$ with $\mathcal{E}\left(x_{n}\right)=0$ for all $n \geq 1$, then there exist three independent sequences $\left(a_{n}\right)_{n \geq 1} \in \bigcap_{1<p<2} \mathcal{R}_{p}^{d}(\mathcal{M}),\left(b_{n}\right)_{n \geq 1} \in \bigcap_{1<p<2} \mathcal{R}_{p}^{c}(\mathcal{M})$, and $\left(c_{n}\right)_{n \geq 1} \in \bigcap_{1<p<2} \mathcal{R}_{p}^{r}(\mathcal{M})$ such that: 
(i) $x_{n}=a_{n}+b_{n}+c_{n}, n \geq 1$,

(ii) for every $1<p<2$, the following inequality holds:

$$
\left\|\left(a_{n}\right)\right\|_{\mathcal{R}_{p}^{d}}+\left\|\left(b_{n}\right)\right\|_{\mathcal{R}_{p}^{c}}+\left\|\left(c_{n}\right)\right\|_{\mathcal{R}_{p}^{r}} \leq \kappa_{p}^{\prime}\left\|\sum_{n \geq 1} x_{n}\right\|_{p} .
$$

\section{Concluding remarks and open problems}

We recall two results about $\Phi$-moment analogue of the Burkholder-Gundy inequalities.

Theorem 6.1 ([13, Corollary 3.3]). If $1<p_{\Phi} \leq q_{\Phi}<\infty$, then there exists a constant $C_{\Phi}$, depending only on $\Phi$, such that for any $x \in L_{\Phi}(\mathcal{M})$,

$$
\tau[\Phi(|x|)] \leq C_{\Phi} \max \left\{\tau\left[\Phi\left(\left(\sum_{n \geq 1}\left|d x_{k}\right|^{2}\right)^{1 / 2}\right)\right], \tau\left[\Phi\left(\left(\sum_{n \geq 1}\left|d x_{k}^{*}\right|^{2}\right)^{1 / 2}\right)\right]\right\} .
$$

Theorem 6.2 ([19, Theorem 7.2]). If $\Phi$ is $p$-convex for some $1<p<2$ and 2-concave, then for every $x \in L_{\Phi}(\mathcal{M})$,

$$
\tau[\Phi(|x|)] \simeq_{\Phi} \inf \left\{\tau\left[\Phi\left(\left(\sum_{n \geq 1}\left|d y_{k}\right|^{2}\right)^{1 / 2}\right)\right]+\tau\left[\Phi\left(\left(\sum_{n \geq 1}\left|d z_{k}^{*}\right|^{2}\right)^{1 / 2}\right)\right]\right\},
$$

where the infimum is taken over all decompositions $x_{n}=y_{n}+z_{n}$ with $\left(y_{n}\right)$ and $\left(z_{n}\right)$ are martingales.

Two natural questions that arise from these results are whether their conditioned versions hold. More precisely, we may ask the following problems:

Problem 6.3. Does the second inequality in Theorem 4.4 hold under the weaker condition $1<p_{\Phi} \leq q_{\Phi}<\infty$ ?

Problem 6.4. Does Theorem 4.1 remain valid under the weaker assumption that $\Phi$ is $p$-convex for some $1<p<2$ and 2-concave?

As far as we know these questions are still open even for the particular case of independent sequences. First, we should note that the restriction $q_{\Phi}<2$ in Theorem 4.1 is due to our use of the simultaneous decompositions stated in Theorem 2.5. Also, at the time of this writing, it is unclear if Proposition 3.10 remain true if we merely assume that $\Phi$ is $q$-concave in place of $q_{\Phi}<q$.

In light of recent developments on theory of noncommutative maximal functions initiated in [20,39], it would be desirable to have the exact noncommutative analogue of (1.2) by replacing the diagonal term $\sum_{n \geq 1} \tau\left[\Phi\left(\left|d x_{n}\right|\right)\right]$ in the statement of Theorem 4.4 by an appropriate " $\Phi$-moment" maximal function term. Such noncommutative maximal functions associated with Orlicz functions were already considered in [2, Definition 3.2] as follows:

$$
\tau\left[\Phi\left(\sup _{n}^{+} d x_{n}\right)\right]:=\inf \left\{\frac{1}{2}\left(\tau\left[\Phi\left(|a|^{2}\right)\right]+\tau\left[\Phi\left(|b|^{2}\right)\right]\right) \sup _{n}\left\|y_{n}\right\|_{\infty}\right\}
$$

where the infimum is taken over all decompositions $d x_{n}=a y_{n} b$ for $a, b \in L_{0}(\mathcal{M})$ and $\left(y_{n}\right) \subset \mathcal{M}$ with $|a|^{2},|b|^{2} \in$ $L_{\Phi}(\mathcal{M})$ and $\sup _{n}\left\|y_{n}\right\|_{\infty} \leq 1$. The following problem is still open.

Problem 6.5. Assume that $2<p_{\Phi} \leq q_{\Phi}<\infty$ and $x \in L_{\Phi}(\mathcal{M})$. Do we have

$$
\tau[\Phi(|x|)] \simeq_{\Phi} \max \left\{\tau\left[\Phi\left(\sup _{n}^{+} d x_{n}\right)\right], \tau\left[\Phi\left(s_{c}(x)\right)\right], \tau\left[\Phi\left(s_{r}(x)\right)\right]\right\} ?
$$


As shown in [25], the answer to the above problem is positive for the case of $p$ th moments when $p \geq 2$. By duality, the corresponding result involving some versions of $\ell_{1}$-valued noncommutative $L_{p}$-spaces is also known for the case $1<p<2$. To this end, we recall that for $1 \leq p<2$ and $1 / p=1 / 2+1 / q$, a sequence $x=\left(x_{n}\right)_{n \geq 1}$ belongs to the space $L_{p}\left(\mathcal{M} ; \ell_{1}^{c}\right)$ if there exist $b_{k, n} \in L_{2}(\mathcal{M})$ and $a_{k, n} \in L_{q}(\mathcal{M})$ such that $x_{n}=\sum_{k} b_{k, n}^{*} a_{k, n}$ for all $n \geq 1$, $\sum_{k, n}\left|b_{n, k}\right|^{2} \in L_{1}(\mathcal{M})$, and $\sum_{k, n}\left|a_{k, n}\right|^{2} \in L_{q / 2}(\mathcal{M})$. The space $L_{p}\left(\mathcal{M} ; \ell_{1}^{c}\right)$ is equipped with the norm

$$
\|x\|_{L_{p}\left(\mathcal{M} ; \ell_{1}^{c}\right)}=\inf \left\{\left(\sum_{k, n \geq 1}\left\|b_{k, n}\right\|_{2}^{2}\right)^{1 / 2}\left\|\left(\sum_{k, n \geq 1}\left|a_{k, n}\right|^{2}\right)^{1 / 2}\right\|_{q}\right\},
$$

where the infimum is taken over all factorizations of the $x_{n}$ 's above. Let $\mathrm{h}_{p}^{1_{c}}(\mathcal{M})$ be the subspace of $L_{p}\left(\mathcal{M} ; \ell_{1}^{c}\right)$ consisting of martingale difference sequences. We refer to [22] for more in depth treatment of $h_{p}^{1_{c}}(\mathcal{M})$. One of the basic properties of $h_{p}^{1_{c}}(\mathcal{M})$ is that it embeds into the diagonal space $\mathrm{h}_{p}^{d}(\mathcal{M})$. Thus, the following result of Junge and Perrin is an improvement of the noncommutative Burkholder inequalities:

Theorem 6.6 ([22, Theorem 5.7]). For $1 \leq p<2$,

$$
\mathcal{H}_{p}^{c}(\mathcal{M})=\mathrm{h}_{p}^{1_{c}}(\mathcal{M})+\mathrm{h}_{p}^{c}(\mathcal{M}) .
$$

The above theorem is a column version that improved [25, Remark 4.8]. As dual to Problem 6.5, it would be interesting to prove a $\Phi$-moment extension of the above result. We believe that such $\Phi$-moment inequalities should lead to a positive solution of Problem 6.5. A first step towards this direction would be to prove if the decomposition in Theorem 6.6 can be achieved simultaneously in the style of Theorem 2.5.

A new development that deserves attention is the noncommutative martingales with continuous filtrations. The theory of Hardy spaces $\mathcal{H}_{p}(\mathcal{M})$ and $\mathrm{h}_{p}(\mathcal{M})$ along with other related spaces that are associated with continuous filtrations have been thoroughly studied by Junge and Perrin in [22]. All known results from the discrete case prior to [22] were extended there to the continuous case and in some cases were improved as witnessed by Theorem 6.6. The natural evolution of this theory would be to push the ideas of Junge and Perrin and develop versions of Hardy spaces associated with noncommutative symmetric spaces and $\Phi$-moments to the general case of continuous filtrations. This is beyond the scope of this paper.

We conclude this paper by recalling that the original proofs of noncommutative Burkholder inequalities by Junge and $\mathrm{Xu}$ were given for martingales in the general $L_{p}$-spaces associated with type III von Neumann algebras ([23]). As is well-known no general theory of noncommutative symmetric spaces is available for type III von Neumann algebras. However, in [30], a theory of Orlicz spaces has been developed for type III von Neumann algebras in the spirit of the construction of the Haagerup $L_{p}$-spaces. Another interesting future direction would be to develop a $\Phi$-moment theory for the type III-case using [30].

\section{References}

[1] T. Bekjan and Z. Chen. Interpolation and $\Phi$-moment inequalities of noncommutative martingales. Probab. Theory Related Fields 152 (1-2) (2012) 179-206. DOI:10.1007/s00440-010-0319-2. MR2875756

[2] T. Bekjan, Z. Chen and A. Osȩkowski. Noncommutative maximal inequalities associated with convex functions. Trans. Amer. Math. Soc. 369 (1) 2017 409-427. DOI:10.1007/s11117-016-0410-8. MR3557778

[3] C. Bennett. Banach function spaces and interpolation methods. I. The abstract theory. J. Funct. Anal. 17 (1974) 409-440. MR0361826

[4] C. Bennett and R. Sharpley. Interpolation of Operators. Academic Press Inc., Boston, MA, 1988. MR0928802

[5] J. Bergh and J. Löfström. Interpolation Spaces. An Introduction. Grundlehren der Mathematischen Wissenschaften 223. Springer-Verlag, Berlin, 1976. MR0482275

[6] D. L. Burkholder. Distribution function inequalities for martingales. Ann. Probab. 1 (1973) 19-42. MR0365692

[7] D. L. Burkholder, B. J. Davis and R. F. Gundy. Integral inequalities for convex functions of operators on martingales. In Proceedings of the Sixth Berkeley Symposium on Mathematical Statistics and Probability (Univ. California, Berkeley, Calif., 1970/1971), Vol. II: Probability Theory 223-240. Univ. California Press, Berkeley, Calif., 1972. MR0400380

[8] D. L. Burkholder and R. F. Gundy. Extrapolation and interpolation of quasi-linear operators on martingales. Acta Math. 124 (1970) $249-304$. MR0440695

[9] P. Butzer and H. Berens. Semi-Groups of Operators and Approximation. Die Grundlehren der mathematischen Wissenschaften 145. SpringerVerlag New York Inc., New York, 1967. MR0230022 
[10] J. Diestel. Sequences and Series in Banach Spaces. Graduate Text in Mathematics 92. Springer-Verlag, New York, 1984. MR0737004

[11] S. Dirksen. Weak-type interpolation for noncommutative maximal operators. J. Operator Theory 73 (2) (2015) 515-532. DOI:10.7900/jot. 2014mar12.2022. MR3346135

[12] S. Dirksen, B. de Pagter, D. Potapov and F. Sukochev. Rosenthal inequalities in noncommutative symmetric spaces. J. Funct. Anal. 261 (10) (2011) 2890-2925. DOI:10.1016/j.jfa.2011.07.015. MR2832586

[13] S. Dirksen and E. Ricard. Some remarks on noncommutative Khintchine inequalities. Bull. Lond. Math. Soc. 45 (3) (2013) 618-624. DOI:10.1112/blms/bds107. MR3065031

[14] P. G. Dodds, T. K. Dodds and B. de Pagter. Noncommutative Banach function spaces. Math. Z. 201 (1989) 583-597. MR1004176

[15] P. G. Dodds, T. K. Dodds and B. de Pagter. Fully symmetric operator spaces. Integral Equations Operator Theory 15 (6) (1992) $942-972$. DOI:10.1007/BF01203122. MR1188788

[16] T. Fack and H. Kosaki. Generalized s-numbers of $\tau$-measurable operators. Pacific J. Math. 123 (1986) 269-300. MR0840845

[17] A. M. Garsia. On a convex function inequality for martingales. Ann. Probab. 1 (1) (1973) 171-174. MR0346897

[18] Y. Jiao, F. Sukochev, G. Xie and D. Zanin $\Phi$-Moment inequalities for independent and freely independent random variables. J. Funct. Anal. 270 (12) (2016) 4558-4596. DOI:10.1016/j.jfa.2016.02.001. MR3490777

[19] Y. Jiao, F. Sukochev, D. Zanin and D. Zhou Johnson-Schechtman inequalities for noncommutative martingales. Preprint, 2015.

[20] M. Junge. Doob's inequality for non-commutative martingales. J. Reine Angew. Math. 549 (2002) 149-190. MR1916654

[21] M. Junge, J. Parcet and Q. Xu. Rosenthal type inequalities for free chaos. Ann. Probab. 35 (4) (2007) 1374-1437. DOI:10.1214/ 009117906000000962. MR2330976

[22] M. Junge and M. Perrin. Theory of $\mathcal{H}_{p}$-spaces for continuous filtrations in von Neumann algebras. Astérisque 362 (2014) vi +134. MR3241706

[23] M. Junge and Q. Xu. Noncommutative Burkholder/Rosenthal inequalities. Ann. Probab. 31 (2) (2003) 948-995. MR1964955

[24] M. Junge and Q. Xu. On the best constants in some non-commutative martingale inequalities. Bull. Lond. Math. Soc. 37 (2) (2005) $243-253$. MR2119024

[25] M. Junge and Q. Xu. Noncommutative Burkholder/Rosenthal inequalities. II. Applications. Israel J. Math. 167 (2008) 227-282. DOI:10.1007/ s11856-008-1048-4. MR2448025

[26] M. Junge and Q. Zeng. Noncommutative Bennett and Rosenthal inequalities. Ann. Probab. 41 (6) (2013) 4287-4316. DOI:10.1214/12AOP771. MR3161475

[27] N. Kalton and S. Montgomery-Smith. Interpolation of Banach spaces. In Handbook of the Geometry of Banach Spaces, Vol. 2 1131-1175. North-Holland, Amsterdam, 2003. MR1999193

[28] N. J. Kalton and F. A. Sukochev. Symmetric norms and spaces of operators. J. Reine Angew. Math. 621 (2008) 81-121. DOI:10.1515/ CRELLE.2008.059. MR2431251

[29] M. A. Krasnosel'skiǐ and J. B. Rutickiř. Convex Functions and Orlicz Spaces. P. Noordhoff Ltd., Groningen, 1961. Translated from the first Russian edition by Leo F. Boron. MR0126722

[30] L. E. Labuschagne. A crossed product approach to Orlicz spaces. Proc. Lond. Math. Soc. (3) 107 (5) (2013) 965-1003. DOI:10.1112/ plms/pdt006. MR3126389

[31] J. Lindenstrauss and L. Tzafriri. Classical Banach Spaces. II: Function Spaces. Springer-Verlag, Berlin, 1979. MR0540367

[32] F. Lust-Piquard. Inégalités de Khintchine dans $C_{p}(1<p<\infty)$. C. R. Math. Acad. Sci. Paris, Sér. I 303 (1986) 289-292. MR0859804

[33] F. Lust-Piquard and G. Pisier. Noncommutative Khintchine and Paley inequalities. Ark. Mat. 29 (1991) 241-260. MR1150376

[34] L. Maligranda. Indices and interpolation. Dissertationes Math. (Rozprawy Mat.) 234 (1985) 49. MR0820076

[35] L. Maligranda. Orlicz Spaces and Interpolation. Seminários de Matemática [Seminars in Mathematics] 5. Universidade Estadual de Campinas, Departamento de Matemática, Campinas, 1989. MR2264389

[36] J. Mogyoródi. On an inequality of H. P. Rosenthal. Period. Math. Hungar. 8 (3-4) (1977) 275-279. MR0488275

[37] E. Nelson. Notes on non-commutative integration. J. Funct. Anal. 15 (1974) 103-116. MR0355628

[38] J. Parcet and N. Randrianantoanina. Gundy's decomposition for non-commutative martingales and applications. Proc. Lond. Math. Soc. (3) 93 (1) (2006) 227-252. MR2235948

[39] G. Pisier. Non-commutative vector valued $L_{p}$-spaces and completely $p$-summing maps. Astérisque 247 (1998) vi +131. MR1648908

[40] G. Pisier and Q. Xu. Non-commutative martingale inequalities. Comm. Math. Phys. 189 (1997) 667-698. MR1482934

[41] G. Pisier and Q. Xu. Non-commutative $L^{p}$-spaces. In Handbook of the Geometry of Banach Spaces, Vol. 2 1459-1517. North-Holland, Amsterdam, 2003. MR1999201

[42] N. Randrianantoanina. Non-commutative martingale transforms. J. Funct. Anal. 194 (2002) 181-212. MR1929141

[43] N. Randrianantoanina. A weak type inequality for non-commutative martingales and applications. Proc. Lond. Math. Soc. (3) 91 (2) (2005) 509-542. MR2167096

[44] N. Randrianantoanina. Conditioned square functions for noncommutative martingales. Ann. Probab. 35 (3) (2007) 1039-1070. DOI:10.1214/ 009117906000000656 . MR2319715

[45] N. Randrianantoanina and L. Wu. Martingale inequalities in noncommutative symmetric spaces. J. Funct. Anal. 269 (7) (2015) $2222-2253$. DOI:10.1016/j.jfa.2015.05.017. MR3378874

[46] H. P. Rosenthal. On the subspaces of $L^{p}(p \geq 2)$ spanned by sequences of independent random variables. Israel J. Math. 8 (1970) $273-303$. MR0271721

[47] F. Sukochev and D. Zanin. Johnson-Schechtman inequalities in the free probability theory. J. Funct. Anal. 263 (10) (2012) $2921-2948$. DOI:10.1016/j.jfa.2012.07.012. MR2973330

[48] M. Takesaki. Theory of Operator Algebras. II: Operator Algebras and Non-commutative Geometry, 6. Encyclopaedia of Mathematical Sciences 125. Springer-Verlag, Berlin, 2003. DOI:10.1007/978-3-662-10451-4. MR1943006

[49] Q. Xu. Analytic functions with values in lattices and symmetric spaces of measurable operators. Math. Proc. Cambridge Philos. Soc. 109 (1991) 541-563. MR1094753 